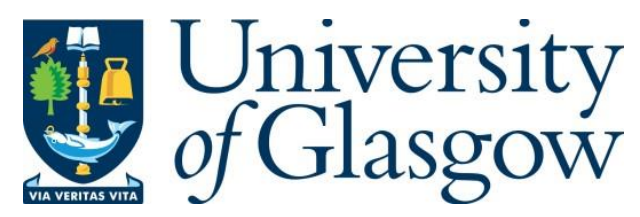

Yang, Y.-F., Lee, Y.-C., Lo, S., Chung, Y.-N., Hsieh, Y.-C., Chiu, W.-C. and Yuan, S.S. F. (2018) A positive feedback loop of IL-17B-IL-17RB activates ERK/ $\beta$-catenin to promote lung cancer metastasis. Cancer Letters, 422, pp. 44-55.

There may be differences between this version and the published version. You are advised to consult the publisher's version if you wish to cite from it.

http://eprints.gla.ac.uk/158903/

Deposited on: 17 April 2018

Enlighten - Research publications by members of the University of Glasgow http://eprints.gla.ac.uk 


\section{A Positive Feedback Loop of IL-17B-IL-17RB Activates ERK/ $\beta$-catenin to Promote Lung Cancer Metastasis}

Yi-Fang Yang ${ }^{\mathrm{a}}$, Yi-Chen Lee ${ }^{\mathrm{a}, \mathrm{b}}$, Steven Lo ${ }^{\mathrm{a}, \mathrm{c}}$, Yi-Ning Chung ${ }^{\mathrm{a}}$, Ya-Ching Hsieh ${ }^{\mathrm{d}}$, Wen-Chin Chiu ${ }^{\mathrm{e}}$, Shyng-Shiou F. Yuan ${ }^{\mathrm{a}, \mathrm{f}, \mathrm{g} *}$

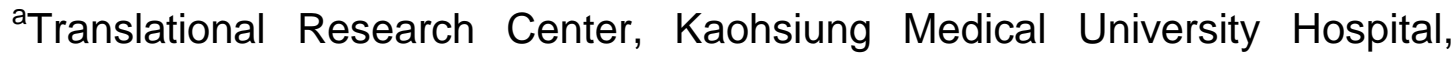
Kaohsiung Medical University, Kaohsiung, Taiwan.

${ }^{\mathrm{b}}$ Department of Anatomy, School of Medicine, College of Medicine, Kaohsiung Medical University, Kaohsiung, Taiwan.

${ }^{\circ}$ College of Medical, Veterinary and Life Sciences, University of Glasgow, Glasgow, UK.

${ }^{d}$ Institute of Cancer Sciences, University of Glasgow, Glasgow, UK.

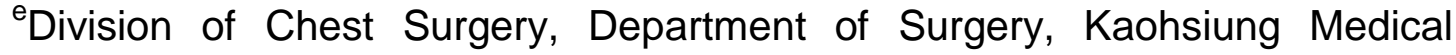
University Hospital, Kaohsiung Medical University, Kaohsiung, Taiwan

fDepartment of Medical Research and Department of Obstetrics and Gynecology, Kaohsiung Medical University Hospital, Kaohsiung Medical University, Kaohsiung, Taiwan.

${ }^{9}$ School of Medicine, College of Medicine, Kaohsiung Medical University, Kaohsiung, Taiwan 
${ }^{*}$ Corresponding author

Shyng-Shiou F. Yuan, Translational Research Center, Kaohsiung Medical University Hospital, Kaohsiung Medical University, 100 Zihyou 1st Road, San-Ming District, Kaohsiung, Taiwan. E-mail: yuanssf@ms33.hinet.net ; TEL: +886-7-3121101\#2557; FAX: +886-7-3112497

Conflict of interest: None 


\section{Abstract}

Inflammation contributes to the development and progression of cancer. Interleukin-17 (IL-17) is an inflammatory cytokine that functions in inflammation and cancer, as well as several other cellular processes. In this study, we investigated the roles and the prognostic value of IL-17 and the IL-17 receptor (IL-17R) in lung cancer. Gene expression microarray analysis followed by Kaplan-Meier survival curve showed that IL-17B was associated with poor patient survival, and IL-17B receptor (IL-17RB) was up-regulated in lung cancer tissue compared with normal tissue. Expression of IL-17RB was associated with lymph node metastasis and distant metastasis, as well as poor patient survival. IL-17RB overexpression significantly increased cancer cell invasion/migration and metastasis in vitro and in vivo. IL-17RB induced ERK phosphorylation, resulting in GSK3 $\beta$ inactivation and leading to $\beta$-catenin up-regulation. IL-17RB also participated in IL-17B synthesis via the ERK pathway. IL-17RB activation is required for IL-17B-mediated ERK phosphorylation. Taken together, IL-17B-IL-17RB signaling and ERK participate in a positive feedback loop that enhances invasion/migration ability in lung cancer cell lines. IL-17RB may therefore serve as an independent prognostic factor and a therapeutic target for lung cancer. 


\section{Keywords}

IL-17RB; IL-17B; ERK; lung cancer; metastasis

\section{Abbreviations}

IL-17RB- IL-17B receptor, IL-17B- Interleukin 17B, ERK- Mitogen-Activated

Protein Kinase 1, GSK3ß-Glycogen Synthase Kinase 3 Beta, NSCLCs-non-small cell lung cancers, COPD-chronic obstructive pulmonary disease, EMT-Epithelial-Mesenchymal Transition, shRNA-short hairpin RNA 


\section{Introduction}

Lung cancer is associated with high mortality worldwide, and more than $80 \%$ of lung cancer cases are non-small cell lung cancers (NSCLCs) [15, 35]. Therefore, it is critical for researchers to identify prognostic factors and therapeutic targets for lung cancer. Accumulating evidence shows that a strong association exists between chronic inflammation and tumorigenesis in different types of cancers, including lung cancer [2, 7, 10]. Moreover, lung diseases, including chronic obstructive pulmonary disease (COPD), idiopathic pulmonary fibrosis, tobacco smoke, air pollutants, pulmonary infections and occupational dust, are all associated with lung cancer, suggesting chronic inflammation plays an important role in the pathogenesis of lung cancer $[7,10$, $27,28]$.

The interleukin receptor family is distinct from other single-transmembrane domain protein families and, upon interleukin binding, functions in multiple cellular processes, including inflammation, immunity and cancer [24]. In particular, the interleukin 17 (IL-17) family (IL-17A to IL-17F) and its distinct cytokine receptor family (IL-17Rs, including IL-17RA to IL-17RE) are important for normal host immune responses and the pathogenesis of human diseases, including airway inflammation and cancer [18, 19, 23, 38, 46]. 
High IL-17 levels have been observed in multiple cancers, including colorectal cancer [22], hepatocellular carcinoma [41], breast cancer [4, 49], ovarian cancer [21], bladder cancer [37], prostate cancer [31], and lung cancer [5]. Furthermore, IL-17 promotes tumor development by inducing the suitable microenvironments at tumor sites by recruiting myeloid-derived suppressor cells [14]. Loss of IL-17 in mice is associated with reduced Stat3 signaling and reduced tumorigenesis [37].

The interactions between IL-17 ligands and receptors are complicated. IL-17A and IL-17B are the ligands for IL-17RA and IL-17RB, respectively [32, 45]. Furthermore, IL-17A binds to IL-17RA/LL-17RC with relatively higher affinity than IL-17F, and IL-17E (IL-25) may have a higher affinity for IL-17RB than IL-17B $[23,24,39,45]$. IL-17R expression is up-regulated in the stimulated, inflamed tissues of patients with bowel diseases and various cancers $[13,32,43]$, while IL-17E is secreted by non-malignant mammary epithelial cells and induces breast cancer apoptosis through IL-25R, an IL-17RB/IL-17RA heterodimer [9, 30]. In addition, high IL-17RB expression is correlated with a poor prognosis in patients with breast cancer $[9,16]$. Accumulating evidence suggests that IL-17 plays important roles in cancer progression; however, the role of its receptor in lung cancer remains unclear. 
In this study, we investigated whether IL-17 ligands and receptors regulated malignant phenotypes in lung cancer. We evaluated the relationship between IL-17 and patient survival by kaplan-merier plotter and also examined the expression levels of IL-17 receptors by CDNA microarray. According to the bioinformatical analysis, we identified the targets and then provided biological studies for verification. We focused on IL-17RB, the receptor responsible for the effects of IL-17B signaling on cancer progression, since a previous study showed that blocking IL-17RB inhibited the invasion and metastatic ability in cancer cells [40]. 


\section{Materials and Methods}

\section{Kaplan-Meier analysis of IL-17 family}

Kaplan-Meier analysis of overall survival was performed by using publicly available lung cancer microarray datasets (sample from 866 patients with adenocarcinoma and 675 patients with squamous cell carcinoma), stratified according to the expression of IL-17A, IL-17B, IL-17C, IL-17D, IL-17E and IL-17F. The publicly available online lung cancer microarray datasets were used for real-time validation of biomarkers related to patients survival (http://kmplot.com/analysis/index.php?p=service\& cancer=lung) [12].

\section{Tissue sample collection}

Paired cancer tissue samples and adjacent non-cancerous lung tissue samples were collected from 139 newly diagnosed lung cancer patients who were surgically treated at Kaohsiung Medical University Hospital from 2007 to 2013 (sample from 105 patients with adenocarcinoma and 34 patients with squamous cell carcinoma). This study was approved by the Institutional Review Board of Kaohsiung Medical University Hospital (KMUHIRB-E(II)-20170140), and written informed consent was obtained from each patient who participated in the study. Staging was performed according 
to the American Joint Committee on Cancer (AJCC) tumor-node-metastasis (TNM) staging system [29]. Disease-free survival (DFS) was defined as the period between the date of surgery and the date of recurrence, and overall survival (OS) was defined as the period between the date of surgery and the date of the patient's death from cancer.

\section{Immunohistochemistry}

Immunohistochemistry $(\mathrm{IHC})$ was performed with a fully automated Bond-Max System (Leica Microsystems, Wetzlar, Germany). All staining steps were performed by the automated instrument, according to the manufacturer's instructions (Leica Microsystems). The primary antibodies used for this experiment are shown in Supplementary Table 7.

\section{Evaluation of immunohistochemical staining}

IL-17RB expression was scored on a scale ranging from 0 to 4 , according to the percentage of positively stained cells $(0$, negative; $1, \leq 25 \%$ of cells stained positive; $2,26-50 \%$ of cells stained positive; $3,51-75 \%$ of cells stained positive; and $4, \geq 76 \%$ of cells stained positive). The score for each specimen was determined by two independent experts simultaneously and 
under the same conditions. Snail expression was scored on a scale ranging from $0-3$, according to the percentage of positively stained cells $(0$, negative; 1 , $\leq 30 \%$ of cells stained positive; $2,31-50 \%$ of cells stained positive; and 3 , $\geq 51 \%$ of cells stained positive). Similarly, Twist expression was scored on a scale ranging from $0-3$, according to the percentage of positively stained cells ( 0 , negative; $1, \leq 50 \%$ of cells stained positive; $2,51-75 \%$ of cells stained positive; and $3, \geq 76$ of cells stained positive).

\section{Cell lines and cell culture conditions}

The lung cancer cell lines CL1-0, CL1-5, H441, H460 and H520 were cultured in RPMI-1640 medium (containing 10\% FBS and 1\% PSG). The A549 and $\mathrm{H} 1299$ cell lines were maintained in DMEM-F12 and DMEM with 10\% FBS and $1 \%$ PSG, respectively.

\section{Reagents}

PD98059 (mitogen-activated protein kinase inhibitor, \#P215) was purchased from Sigma (St. Louis, MO, USA). U-46619 (activator of ERK1 and ERK2, \#sc-201242) was purchased from Santa Cruz Biotechnology (Dallas, TX, USA). The Proteome ProfilerTM Human Phospho-kinase Array Kit 
(ARY003B) and recombinant human IL-17B (1248-IB-025/CF) were purchased from R\&D Systems.

\section{Lentiviral infection}

The negative control lentiviral expression vector (LPP-NEG-Lv-105-025) and the lentiviral expression vector containing IL-17RB (LPP-Z1990-Lv105-200-S) were purchased from GeneCopoeia ${ }^{\text {TM }}$. The pLKO-.1-shLuc967, shIL-17RB (TRC58814, TRC58816) and shlL-17B (TRC8595, TRC8596) lentiviral shRNA supernatants were purchased from National RNAi Core Facility (Taipei, Taiwan).

\section{Western blot analysis}

Total protein $(50 \mu \mathrm{g})$ was extracted from the above cell lines and loaded onto a $10 \%$ SDS-PAGE gel. After electrophoresis, the proteins were transferred to a PVDF membrane, which was blocked with 5\% noon-fat milk in TBST. Western blotting was performed using the appropriate antibodies (Supplementary Table 7).

\section{Growth curve assay}


Cells $\left(2000\right.$ cells/well) were seeded in a 96 -well plate and incubated at $37^{\circ} \mathrm{C}$ with $5 \% \mathrm{CO}_{2}$ for 24 to $72 \mathrm{~h}$. The cell growth curve was determined by 3-(4,5-dimethylthiazol-2-yl)-2,5-diphenyltetrazolium bromide (MTT) assay.

\section{Cell migration and invasion assays}

Cell migration and invasion assays were performed with a Falcon ${ }^{\mathrm{TM}}$ HTS Multiwell Insert System (8- $\mu$ m pores, BD Biosciences, San Jose, CA). For invasion assay, the upper chamber of each multiwell insert (transwell) was precoated with Matrigel (BD Biosciences, San Jose, CA), and medium containing $10 \%$ FBS was added to each 24 -well plate. The cells were then resuspended in serum-free medium $\left(2 \times 10^{5} / \mathrm{mL}\right)$ and loaded into the upper chamber of the transwell in $100 \mu \mathrm{L}$ aliquots. After $18-24 \mathrm{~h}$, the cells that had migrated to the lower chamber were stained by crystal violet and counted under a light microscope (entire well). All experiments were performed in quadruplicate. The procedure for the migration assay was similar to that for the invasion assay; however, the upper chamber of the transwell was not coated with Matrigel for the migration assay. To block IL-17B, we incubated IL-17RB-overexpressing cells with an IL-17B-neutralizing antibody $(4 \mu \mathrm{g} / \mathrm{mL})$ for $48 \mathrm{~h}$. Specifically, the cells were trypsinized for the transwell assay after 
incubation, and an IL-17B-neutralizing antibody was added to the upper chamber of the transwell.

\section{Animal studies}

The animal experiments were conducted in accordance with a protocol approved by the Use Committee of Kaohsiung Medical University. Six-week-old male immunodeficient mice (BALB/cAnN.Cg-Foxn1nu/CrINarl) were purchased from the National Laboratory Animal Center (NLAC, Taiwan). For in vivo metastasis assay, cells were resuspended in PBS $\left(1 \times 10^{6}\right.$ cells $/ 100$ $\mu \mathrm{L} \mathrm{PBS} /$ mouse) before being injected into each mouse via the tail vein. Five weeks after tail vein injection, all mice were sacrificed and the numbers of lung nodules were quantified by a dissecting microscope after the appropriate tissues were stained with hematoxylin and eosin (H\&E).

\section{Enzyme-linked Immunosorbent Assay (ELISA)}

The cells were plated in 6-well plates and incubated at $37^{\circ} \mathrm{C}$ for $24 \mathrm{~h}$, and then the medium was replaced with fresh medium, and the cells were incubated at $37^{\circ} \mathrm{C}$ for an additional $24 \mathrm{~h}$. The conditioned medium was then collected, and IL-17B expression was determined by a human IL-17B duo-set 
ELISA kit (DY1248, R\&D Systems).

\section{Real time-PCR}

The stable cell lines CL1-0/vector control and CL1-0/LL-17RB $\left(3 \times 10^{5}\right.$ cells/dish) were separately plated in 6-cm culture plates and incubated overnight at $37^{\circ} \mathrm{C}$ with $5 \% \mathrm{CO}_{2}$. The $\mathrm{CL} 1-0 / \mathrm{LL}-17 \mathrm{RB}$ cells were then treated with a MEK/ERK inhibitor for $24 \mathrm{~h}$, followed by total RNA extraction for real-time PCR analysis (SYBR system). The following primers were used for this experiment: $I L-17 B$, forward: 5'TCAACTTGCAGCTGTGGATGTC-3' and reverse: 5'GGTCCTCCTGCATGGTGAAG-3'; and GAPDH, forward: 5'GCACCACCAACTGCTTAGCA-3' and reverse: 5'TCTTCTGGGTGGCA GTGATG-3'.

\section{Statistical analysis}

All statistical analyses were performed using SPSS (Statistical Package for the Social Sciences, version 19) software. Tissues with IL-17RB expression scores of 1 and 2 were categorized as having low IL-17RB expression, and tissues with scores of 3 and 4 were categorized as having high IL-17RB expression. Chi-square tests were performed to determine if differences in age 
at diagnosis, sex, tumor grade, tumor size (T stage), the extent of lymph node metastasis ( $\mathrm{N}$ stage), the extent of distant metastasis ( $\mathrm{M}$ stage), patient performance and smoking status existed between the high IL-17RB expression group and the low IL-17RB expression group. Survival curves were generated by the Kaplan-Meier method, and the log rank test was used to evaulate the significance of differences between curves. Hazard ratios (HRs) and $95 \%$ confidence intervals (Cls) were calculated by univariate and multivariable Cox regression models, which were used to investigate the associations between clinicopathological characteristics and patient survival. $P<0.05$ was considered statistically significant. Spearman's correlation test was performed to evaluate the significance of correlations between paramters. Difference between two groups was compared by Student $t$ test. Multiple group settings were compared by Tukey's post-hoc test. Other materials and methods provided in the online supplement. 


\section{Results}

\section{In silico mRNA profiles of IL-17 and the IL-17R in lung cancer}

We evaluated the expression of the IL-17 family in lung cancer with online survival analysis software [12]. High IL17B, IL17C and IL17E expression was significantly associated with worse overall survival. However, IL17A, IL17D and IL17F expression was not significantly associated with worse overall survival (Fig. 1A). Cytokines act on target cells by binding to specific receptors. For example, IL-17B binds to IL-17RB, IL-17C binds to a heterodimer comprising IL-17RA/IL-17RE, and IL-17E binds to a heterodimer comprising IL-17RA/IL-17RB [34]. We subsequently examined the expression profiles of IL-17RA, IL-17RB and IL-17RE in other microarray datasets. Expression of IL-17RA, IL-17RB and IL-17RE in 20 normal lung tissues and the tissues from 226 patients with lung adenocarcinoma (AdC) was compared in the GSE31210 datasets. IL-17RB and IL-17RE expression levels were significantly up-regulated in patients with lung AdC compared with the normal lung tissue specimens (Fig. 1B). We also analyzed IL-17R expression in normal lung tissues and their paired tumor tissues from 5 patients with squamous cell carcinoma (SCC) in the GSE3268 datasets (Supplementary Fig. 1). IL-17RA expression was down-regulated in patients with lung AdC and SCC compared 
with the normal lung tissue specimens. Notably, IL-17RA/IL-17RE heterodimers and IL-17RA/IL-17RB heterodimers are required to facilitate IL-17C- and IL-17E-mediated signaling, respectively [34]. These results suggested that IL-17B and IL-17RB may play an important role in lung cancer progression.

\section{IL-17RB up-regulation is associated with poor clinical outcomes in lung}

\section{cancer}

To study the expression of IL-17RB protein in lung cancer patients, 40 sets of primary lung tumor specimens and paired pulmonary alveolar tissue specimens were analyzed by IHC. IL-17RB was significantly up-regulated in 21 of the 40 lung tumor specimens compared with the pulmonary alveolar tissue specimens from the same patients (Fig. 2A; Supplementary Table 1 and Table 2). To further evaluate IL-17RB protein in lung cancer, we examined its expression in primary lung tumors from 139 patients with lung cancer by IHC and correlated IL-17RB expression with the clinicopathological characteristics of these patients. As shown in Fig. 2B, IL-17RB protein expression in cancer tissues was classified into four quartiles (scores). Tissues with scores of 1 and 2 were categorized as having low IL-17RB 
expression, and tissues with scores of 3 and 4 were categorized as having high IL-17RB expression. We found that high IL-17RB expression levels in lung cancer tissues were significantly associated with positive lymph nodes metastasis ( $\mathrm{N}$ status; $P=0.002$ ) and positive distant metastasis ( $\mathrm{M}$ status; $P=0.03)$ (Table 1).

Survival analysis by the log-rank test demonstrated the decreased disease-free survival (DFS) and overall survival (OS) rates in the high IL-17RB expression group compared with the low IL-17RB expression group $(P<0.001)$ ( Fig. 2C). We also evaluated the risk factors associated with lung cancer. Hazard ratios (HRs) were estimated by univariate and multivariable Cox regression (Fig. 2D; Supplementary Table 3 and Table 4). The univariate and multivariate analyses of DFS and OS showed that IL-17RB expression was a significant predictor of patient outcome (Fig. 2D; Supplementary Table 3 and Table 4).

\section{Up-regulation of IL-17RB expression enhances lung cancer cell invasion/migration and metastasis}

From clinical data analysis, we found that IL-17RB may play an important role in lung cancer metastasis (Table 1). We then evaluated the effect of 
IL-17RB on lung cancer cell invasion and migration. The western blotting results showed that the levels of endogenous IL-17RB were up-regulated in highly invasive CL1-5 and A549 cells, and IL-17RB was positively correlated with invasion ability in 6 human lung cancer cell lines (Fig. 3A-B). Knocking down IL-17RB expression in the highly invasive CL1-5 and A549 lung cancer cell lines significantly reduced invasion/migration ability in these cell lines compared with the shluc control cell line (Fig. 3C; Supplementary Fig. 2A). Conversely, overexpressing IL-17RB in the poorly invasive H441 and CL1-0 cell lines significantly enhanced their invasion/migration ability compared with the control cell lines (Fig. 3D; Supplementary Fig. 2B). Neither knockdown nor overexpression of IL-17RB in lung cancer cells affected the proliferation potential of the cells (Supplementary Fig. 2C).

We next investigated the effect of IL-17RB on metastasis in vivo. We stably overexpressed IL-17RB in H441 cells and intravenously injected the cells into the tail veins of mice. IL-17RB overexpression resulted in a significant increase in the number of metastatic nodules in the lungs of the indicated mice compared with vector control (Fig. 3E). We also quantified the degree of lymph node invasion by analyzing the expression of cytokeratin 18 (CK18), a lymph node metastasis marker [42, 47]. IL-17RB overexpression 
resulted in an increase of CK18 expression in mouse lymph nodes compared with vector control (Fig. 3F).

\section{IL-17RB promotes invasion/migration through activation of ERK}

\section{signaling pathway}

To determine the molecular mechanisms by which IL-17RB regulates cancer cell invasion/migration, we used human phosphokinase arrays to examine control cells (H441/vector) and IL17RB-overexpressing H441 cells (Fig. 4A). The analysis showed that p-ERK1/2, p-GSK3 $\beta(S e r 9), \beta$-catenin, p-AMPK and p-Chk2 expression levels were increased in IL17RB-overexpressing H441 cells compared with control cells (Fig. 4A). We confirmed these results using western blotting, which showed that $p$-ERK1/2, p-GSK3 $\beta$ (Ser9), $\beta$-catenin and $p$-Chk2 expression levels were significantly increased in IL-17RB-overexpressing H441 cells compared with control cells (Fig. 4B). We focused on ERK1/2 in subsequent experiments because it phosphorylates the Ser9 residue of GSK3 $\beta$, leading to $\beta$-catenin up-regulation [8]. We investigated whether IL-17RB promotes invasion/migration via ERK activation. Transwell assay demonstrated that blocking ERK1/2 phosphorylation with an MEK/ERK inhibitor (PD98059) inhibited 
invasion/migration in IL-17RB-overexpressing cells (Fig. 4C).

We next investigated whether IL-17RB expression inactivates GSK3 $\beta$ and up-regulates $\beta$-catenin via the ERK1/2 pathway. As shown in Fig. 4D, IL-17RB-induced phosphorylation of ERK1/2 and GSK3 $\beta$ (Ser9) and expression of $\beta$-catenin were abolished by the MEK/ERK inhibitor PD98059. Similarly, IL-17RB knockdown resulted in reduced phosphorylation of ERK1/2 and GSK3 $\beta$ (Ser9) and expression of $\beta$-catenin (Fig. 4E). These data suggested that IL-17RB enhanced lung cancer cell invasion through the ERK/GSKß/ß-catenin signaling pathway.

\section{IL-17RB up-regulation correlates with Snail/Twist expression in human}

\section{lung cancer}

Activation of ERK pathway has been shown to be associated with epithelial-mesenchymal transition (EMT) [6, 26, 33]. We examined whether IL-17RB expression up-regulates EMT marker expression through the ERK1/2 pathway. The results showed that the expression of Snail and Twist was significantly up-regulated in IL-17RB-overexpressing cells but was decreased in both MEK/ERK inhibitor-treated and IL-17RB-knockdown cells (Fig. 5A-B). We next examined whether IL-17RB expression was correlated with Snail and 
Twist expression in lung cancer tissues. IHC staining for IL-17RB and Snail showed that IL-17RB expression was positively correlated with Snail expression in lung cancer tissues (Spearman nonparametric correlation test; correlation coefficient $=0.247 ; P=0.014 ; \mathrm{n}=79$ ) (Fig. $5 \mathrm{C}$ and 5E; Supplementary Table 5). Similar result was observed between IL-17RB and Twist, as IL-17RB expression was positively correlated with Twist expression in lung cancer tissues (Spearman nonparametric correlation test; correlation coefficient=0.234; $P=0.007 ; \mathrm{n}=109)$ (Fig. 5D and 5F; Supplementary Table 6). Moreover, we analyzed the GSE3141 datasets, which contained data for 111 patients with lung cancer, and obtained results similar to our study (Fig. 5G).

\section{IL-17RB up-regulation mediated IL-17B secretion through the ERK} pathway

In our study, the MEK/ERK inhibitor PD98059 decreased IL-17RB expression in IL-17RB-overexpressing cells (Fig. 4D and 5A). Furthermore, it has been reported that IL-17RB expression is induced by IL-17B in other cell types $[1,16]$. We therefore explored the possibility that IL-17B induces IL-17RB expression in lung cancer cells. IL-17B treatment caused significant IL-17RB up-regulation in CL1-0 and H441 lung cancer cells (Fig. 6A). Also, 
IL-17RB overexpression resulted in the up-regulation of IL-17B expression levels, whereas treatment with the MEK/ERK inhibitor PD98059 down-regulated IL-17B levels in IL-17RB-overexpressing cells (Fig. 6B; Supplementary Fig 3). These results indicated that IL-17RB-ERK signaling may be involved in IL-17B synthesis.

We also investigated whether IL-17B mediated invasion/migration in IL-17RB-overexpressing cells. IL-17RB overexpression-induced migration and invasion activity was suppressed by an IL-17B-neutralizing antibody (Fig. 6C). In addition, IL-17B knockdown resulted in IL-17RB and ERK pathway down-regulation in IL-17RB-overexpressing CL1-0 cells (Fig. 6D). Conversely, treatment with the ERK activator U46619 caused a significant increase in ERK phosphorylation in CL1-0 cells, leading to IL-17B and IL-17RB up-regulation, whereas treatment with the MEK inhibitor caused a decrease in ERK phosphorylation in CL-1-0 cells (Fig. 6E). We subsequently evaluated IL-17B levels in the serum of mice with lung metastasis of IL-17RB-overexpressing cancer cells and found that IL-17B levels were significantly higher in these mice than in vector control-treated mice (Fig. 6F). Furthermore, correlation analysis of $I L-17 B$ and $I L-17 R B$, which was performed using GSE3141 datasets (lung cancer microarray datasets), showed that $I L-17 B$ expression 
was positively correlated with IL-17RB expression (Fig. 6G). Taken together, our results suggest that IL-17RB activation is required for IL-17B-mediated ERK phosphorylation, which leads to up-regulation of $\beta$-catenin, Snail and Twist and contributes to lung cancer metastasis (Fig. 6H). 


\section{Discussion}

In this study, the IL-17 family was assessed in lung cancer patients, with high IL-17B and its receptor IL-17RB identified as poor prognostic factors. IL-17B and IL-17RB were associated with poor survival in patients with lung cancer, with high IL-17RB expression associated with shorter DFS and OS times, indicating that they play an important role in lung cancer progression. Although other members of the IL-17 family have been implicated in tumor metastasis, such as IL-17A mediated endothelial breach promoting lung metastasis [20], we have demonstrated that these ligands are not associated with a poorer prognosis and are not significantly expressed in lung cancer tissues.

IL-17RB overexpression increased migration/invasion activity in noninvasive lung cancer cell lines, whereas IL-17RB knockdown inhibited migration/invasion ability in highly invasive cell lines. Our data demonstrated that increases in IL-17RB-mediated phosphorylation of ERK led to phosphorylation of GSK3 $\beta$, which induced the up-regulation of $\beta$-catenin, a transcription factor in the Wnt pathway [25]. Moreover, the IL-17RB-ERK pathway mediated IL-17B up-regulation in lung cancer cell lines, whereas treatment with IL-17B increased the effects of IL-17RB expression via the ERK 
pathway in lung cancer cells. These findings suggest that IL-17B-IL-17RB signaling and ERK participate in a positive feedback loop to promote lung cancer metastasis (Fig. 6H).

Furthermore, we showed that IL-17RB overexpression promoted invasion/migration activity and increased IL-17B synthesis via the ERK pathway in lung cancer cells. The invasion/migration assay showed that IL-17B blockade inhibited invasion/migration ability in IL-17RB-overexpressing cells (Fig. 6C). Knocking down IL-17B significantly reduced IL-17RB expression and ERK phosphorylation in IL-17RB-overexpressing cells compared with IL-17RB-overexpressing shluc cells (Fig. 6D). Moreover, treatment with IL-17B induced IL-17RB up-regulation in CL1-0 and H441 lung cancer cells, whereas knockdown of IL-17B inhibited IL-17RB expression in IL-17RB-overexpressing cells (Fig. 6A and 6D). Based on these findings, we surmised that IL-17RB is required for IL-17B activation, which results in enhanced invasion/migration in lung cancer cells.

Other mechanistic pathways have been suggested for IL-17, including IL-17B/IL-17RB pro-survival effects in breast cancer cells via TRAF6, NF-kB and BCL-2 mediated anti-apoptosis [16]. IL-17 has also been shown to promote invasion and metastasis via MMP7 induced EMT in prostate cancer 
[48]. One study relevant to lung cancer suggests that IL-17 may promotes lung cancer cell migration/invasion activity by inducing EMT and up-regulating the NF-kB/ZEB1 pathway, but this study fails to distinguish between IL-17 subtypes, and the effects may stem from the less clinically relevant IL-17A form [11]. In agreement with our result (Fig. 1A), Jungnickel et al. also showed that the expression of IL-17C correlates with poor disease outcome in lung cancer, but they did not investigative the role of its receptor in lung cancer [17]. In contrast, our data using the clinically relevant IL-17B subtype demonstrated that EMT in lung cancer may be mediated via Snail and Twist, as IL-17RB overexpression resulted in their up-regulation, whereas IL-17RB knockdown inhibited their expression in lung cancer cell lines.

An early study reported that T-helper type 17 (Th17) cells, a CD4 ${ }^{+} \mathrm{T}$ cell subset, generated IL-17 in response to the cytokines IL-6, TGF- $\beta$, IL-21, IL-1, and IL-23 in autoimmune diseases [36]. Targeting ERK/MAPK signaling with a MEK inhibitor effectively reduces IL-17 synthesis in Th17 cells, whereas treatment with IL-23 and IL-1 $\beta$ increases IL-17 levels in Th17 cells $[3,36]$. Furthermore, treatment with the ERK activator U49919 significantly up-regulated IL-1 $\beta$ and IL-6 in the microglia cell line BV2 [44]. Here, we showed that U49916 treatment increased IL-17B and IL-17RB expression in 
CL1-0 lung cancer cells (Fig. 6E). Moreover, ERK phosphorylation induces IL-17B synthesis in lung cancer cells, a change inhibited by the MEK inhibitor (Fig. 6E). Recent studies also showed that IL-17B treatment or IL-17RB overexpression led to ERK phosphorylation and promoted metastasis whereas treatment with a neutralizing antibody against IL-17RB inhibits metastasis in pancreatic cancer [40]. We could not exclude the possibility that IL-17B is secreted by other cell types; however, this is the first study to demonstrate that IL-17RB mediated IL-17B synthesis via the ERK pathway, providing the basis for a therapeutic target that may reduce lung cancer cell metastasis.

In conclusion, this is the first study to identify clinically relevant subtypes of the IL-17 family through analysis of lung cancer patient tissues - IL-17B and IL-17RB - and delineate their mechanism of action in lung cancer invasion and metastasis. As clear evidence emerges of the role that inflammation plays in cancer progression, IL-17B-IL-17RB has been identified as both a key prognostic marker and ideal target for lung cancer treatment. 


\section{Acknowledgments}

The IL-17RB antibody was kindly provided by Dr. Wen-Hwa Lee from the Institute of Academia Sinica. This work was supported by grants from Kaohsiung Medical University Hospital (KMUH105-5R32), Kaohsiung Medical University (Aim for the Top Journals Grant, KMU-TP105D10, KMU-DT106004) and National Science Council (MOST 106-2314-B-037-005-MY2). The funders had no role in study design, data collection and analysis, decision to publish, or preparation of the manuscript.

\section{Author contributions statement}

The authors contributed in the following way: design and write the manuscripts: Y.-F.Y, Y.-C.L and S.-S.F.Y; provide materials: S.-S.F.Y; perform experiments: Y.-F.Y, Y.-N.C and W.-C.C; interpret data: Y.-F.Y, Y.-C.L, S.L, Y.-C.H and S.-S.F.Y. 


\section{References}

[1] Q. Bie, C. Sun, A. Gong, C. Li, Z. Su, D. Zheng, X. Ji, Y. Wu, Q. Guo, S.

Wang, $\mathrm{H} . \mathrm{Xu}$, Non-tumor tissue derived interleukin-17B activates IL-17RB/AKT/beta-catenin pathway to enhance the stemness of gastric cancer, Scientific reports, 6 (2016) 25447.

[2] D.R. Brenner, J.R. McLaughlin, R.J. Hung, Previous lung diseases and lung cancer risk: a systematic review and meta-analysis, PloS one, 6 (2011) e17479.

[3] C.F. Brereton, C.E. Sutton, S.J. Lalor, E.C. Lavelle, K.H. Mills, Inhibition of ERK MAPK suppresses IL-23- and IL-1-driven IL-17 production and attenuates autoimmune disease, Journal of immunology, 183 (2009) 1715-1723.

[4] W.C. Chen, Y.H. Lai, H.Y. Chen, H.R. Guo, I.J. Su, H.H. Chen, Interleukin-17-producing cell infiltration in the breast cancer tumour microenvironment is a poor prognostic factor, Histopathology, 63 (2013) 225-233.

[5] X. Chen, J. Wan, J. Liu, W. Xie, X. Diao, J. Xu, B. Zhu, Z. Chen, Increased IL-17-producing cells correlate with poor survival and lymphangiogenesis in NSCLC patients, Lung cancer, 69 (2010) 348-354.

[6] L.Y. Chiu, I.L. Hsin, T.Y. Yang, W.W. Sung, J.Y. Chi, J.T. Chang, J.L. Ko, G.T. 
Sheu, The ERK-ZEB1 pathway mediates epithelial-mesenchymal transition in pemetrexed resistant lung cancer cells with suppression by vinca alkaloids, Oncogene, 36 (2017) 242-253.

[7] L.M. Coussens, Z. Werb, Inflammation and cancer, Nature, 420 (2002) 860-867.

[8] Q. Ding, W. Xia, J.C. Liu, J.Y. Yang, D.F. Lee, J. Xia, G. Bartholomeusz, Y. Li, Y. Pan, Z. Li, R.C. Bargou, J. Qin, C.C. Lai, F.J. Tsai, C.H. Tsai, M.C. Hung, Erk associates with and primes GSK-3beta for its inactivation resulting in upregulation of beta-catenin, Molecular cell, 19 (2005) 159-170.

[9] S. Furuta, Y.M. Jeng, L. Zhou, L. Huang, I. Kuhn, M.J. Bissell, W.H. Lee, IL-25 causes apoptosis of IL-25R-expressing breast cancer cells without toxicity to nonmalignant cells, Science translational medicine, 3 (2011) 78ra31. [10] S.I. Grivennikov, M. Karin, Inflammation and oncogenesis: a vicious connection, Current opinion in genetics \& development, 20 (2010) 65-71.

[11] K. Gu, M.M. Li, J. Shen, F. Liu, J.Y. Cao, S. Jin, Y. Yu, Interleukin-17-induced EMT promotes lung cancer cell migration and invasion via NF-kappaB/ZEB1 signal pathway, American journal of cancer research, 5 (2015) 1169-1179.

[12] B. Gyorffy, P. Surowiak, J. Budczies, A. Lanczky, Online survival analysis 
software to assess the prognostic value of biomarkers using transcriptomic data in non-small-cell lung cancer, PloS one, 8 (2013) e82241.

[13] D. Haudenschild, T. Moseley, L. Rose, A.H. Reddi, Soluble and transmembrane isoforms of novel interleukin- 17 receptor-like protein by RNA splicing and expression in prostate cancer, The Journal of biological chemistry, 277 (2002) 4309-4316.

[14] D. He, H. Li, N. Yusuf, C.A. Elmets, J. Li, J.D. Mountz, H. Xu, IL-17 promotes tumor development through the induction of tumor promoting microenvironments at tumor sites and myeloid-derived suppressor cells, Journal of immunology, 184 (2010) 2281-2288.

[15] P.C. Hoffman, A.M. Mauer, E.E. Vokes, Lung cancer, Lancet, 355 (2000) 479-485.

[16] C.K. Huang, C.Y. Yang, Y.M. Jeng, C.L. Chen, H.H. Wu, Y.C. Chang, C. Ma, W.H. Kuo, K.J. Chang, J.Y. Shew, W.H. Lee, Autocrine/paracrine mechanism of interleukin-17B receptor promotes breast tumorigenesis through NF-kappaB-mediated antiapoptotic pathway, Oncogene, 33 (2014) 2968-2977. [17] C. Jungnickel, L.H. Schmidt, L. Bittigkoffer, L. Wolf, A. Wolf, F. Ritzmann, A. Kamyschnikow, C. Herr, M.D. Menger, T. Spieker, R. Wiewrodt, R. Bals, C. Beisswenger, IL-17C mediates the recruitment of tumor-associated neutrophils 
and lung tumor growth, Oncogene, 36 (2017) 4182-4190.

[18] M. Kawaguchi, M. Adachi, N. Oda, F. Kokubu, S.K. Huang, IL-17 cytokine family, The Journal of allergy and clinical immunology, 114 (2004) 1265-1273; quiz 1274 .

[19] J.K. Kolls, A. Linden, Interleukin-17 family members and inflammation, Immunity, 21 (2004) 467-476.

[20] P. Kulig, S. Burkhard, J. Mikita-Geoffroy, A.L. Croxford, N. Hovelmeyer, G. Gyulveszi, C. Gorzelanny, A. Waisman, L. Borsig, B. Becher, IL17A-Mediated Endothelial Breach Promotes Metastasis Formation, Cancer immunology research, 4 (2016) 26-32.

[21] C. Lan, X. Huang, S. Lin, H. Huang, Q. Cai, J. Lu, J. Liu, High density of IL-17-producing cells is associated with improved prognosis for advanced epithelial ovarian cancer, Cell and tissue research, 352 (2013) 351-359.

[22] S. Le Gouvello, S. Bastuji-Garin, N. Aloulou, H. Mansour, M.T. Chaumette, F. Berrehar, A. Seikour, A. Charachon, M. Karoui, K. Leroy, J.P. Farcet, I. Sobhani, High prevalence of Foxp3 and IL17 in MMR-proficient colorectal carcinomas, Gut, 57 (2008) 772-779.

[23] J. Lee, W.H. Ho, M. Maruoka, R.T. Corpuz, D.T. Baldwin, J.S. Foster, A.D. Goddard, D.G. Yansura, R.L. Vandlen, W.I. Wood, A.L. Gurney, IL-17E, a novel 
proinflammatory ligand for the IL-17 receptor homolog IL-17Rh1, The Journal of biological chemistry, 276 (2001) 1660-1664.

[24] T.S. Li, X.N. Li, Z.J. Chang, X.Y. Fu, L. Liu, Identification and functional characterization of a novel interleukin 17 receptor: a possible mitogenic activation through ras/mitogen-activated protein kinase signaling pathway, Cellular signalling, 18 (2006) 1287-1298.

[25] B.T. MacDonald, K. Tamai, X. He, Wnt/beta-catenin signaling: components, mechanisms, and diseases, Developmental cell, 17 (2009) 9-26.

[26] D. Nagarajan, T. Melo, Z. Deng, C. Almeida, W. Zhao, ERK/GSK3beta/Snail signaling mediates radiation-induced alveolar epithelial-to-mesenchymal transition, Free radical biology \& medicine, 52 (2012) 983-992.

[27] D.S. O'Callaghan, D. O'Donnell, F. O'Connell, K.J. O'Byrne, The role of inflammation in the pathogenesis of non-small cell lung cancer, Journal of thoracic oncology : official publication of the International Association for the Study of Lung Cancer, 5 (2010) 2024-2036.

[28] J. Putila, N.L. Guo, Combining COPD with Clinical, Pathological and Demographic Information Refines Prognosis and Treatment Response Prediction of Non-Small Cell Lung Cancer, PloS one, 9 (2014) e100994. 
[29] R. Rami-Porta, J.J. Crowley, P. Goldstraw, The revised TNM staging system for lung cancer, Annals of thoracic and cardiovascular surgery : official journal of the Association of Thoracic and Cardiovascular Surgeons of Asia, 15 (2009) 4-9.

[30] E.A. Rickel, L.A. Siegel, B.R. Yoon, J.B. Rottman, D.G. Kugler, D.A. Swart, P.M. Anders, J.E. Tocker, M.R. Comeau, A.L. Budelsky, Identification of functional roles for both IL-17RB and IL-17RA in mediating IL-25-induced activities, Journal of immunology, 181 (2008) 4299-4310.

[31] K.S. Sfanos, T.C. Bruno, C.H. Maris, L. Xu, C.J. Thoburn, A.M. DeMarzo, A.K. Meeker, W.B. Isaacs, C.G. Drake, Phenotypic analysis of prostate-infiltrating lymphocytes reveals $\mathrm{TH} 17$ and Treg skewing, Clinical cancer research : an official journal of the American Association for Cancer Research, 14 (2008) 3254-3261.

[32] Y. Shi, S.J. Ullrich, J. Zhang, K. Connolly, K.J. Grzegorzewski, M.C. Barber, W. Wang, K. Wathen, V. Hodge, C.L. Fisher, H. Olsen, S.M. Ruben, I. Knyazev, Y.H. Cho, V. Kao, K.A. Wilkinson, J.A. Carrell, R. Ebner, A novel cytokine receptor-ligand pair. Identification, molecular characterization, and in vivo immunomodulatory activity, The Journal of biological chemistry, 275 (2000) 19167-19176. 
[33] B.N. Smith, L.J. Burton, V. Henderson, D.D. Randle, D.J. Morton, B.A.

Smith, L. Taliaferro-Smith, P. Nagappan, C. Yates, M. Zayzafoon, L.W. Chung, V.A. Odero-Marah, Snail promotes epithelial mesenchymal transition in breast cancer cells in part via activation of nuclear ERK2, PloS one, 9 (2014) e104987.

[34] X. Song, Y. Qian, IL-17 family cytokines mediated signaling in the pathogenesis of inflammatory diseases, Cellular signalling, 25 (2013) 2335-2347.

[35] A. Spira, D.S. Ettinger, Multidisciplinary management of lung cancer, The New England journal of medicine, 350 (2004) 379-392.

[36] C.E. Sutton, S.J. Lalor, C.M. Sweeney, C.F. Brereton, E.C. Lavelle, K.H. Mills, Interleukin-1 and IL-23 induce innate IL-17 production from gammadelta T cells, amplifying Th17 responses and autoimmunity, Immunity, 31 (2009) $331-341$.

[37] L. Wang, T. Yi, M. Kortylewski, D.M. Pardoll, D. Zeng, H. Yu, IL-17 can promote tumor growth through an IL-6-Stat3 signaling pathway, The Journal of experimental medicine, 206 (2009) 1457-1464.

[38] J. Witowski, K. Ksiazek, A. Jorres, Interleukin-17: a mediator of inflammatory responses, Cellular and molecular life sciences : CMLS, 61 
(2004) 567-579.

[39] J.F. Wright, F. Bennett, B. Li, J. Brooks, D.P. Luxenberg, M.J. Whitters, K.N. Tomkinson, L.J. Fitz, N.M. Wolfman, M. Collins, K. Dunussi-Joannopoulos, M. Chatterjee-Kishore, B.M. Carreno, The human IL-17F/IL-17A heterodimeric cytokine signals through the IL-17RA/IL-17RC receptor complex, Journal of immunology, 181 (2008) 2799-2805.

[40] H.H. Wu, W.W. Hwang-Verslues, W.H. Lee, C.K. Huang, P.C. Wei, C.L. Chen, J.Y. Shew, E.Y. Lee, Y.M. Jeng, Y.W. Tien, C. Ma, W.H. Lee, Targeting IL-17B-IL-17RB signaling with an anti-IL-17RB antibody blocks pancreatic cancer metastasis by silencing multiple chemokines, The Journal of experimental medicine, 212 (2015) 333-349.

[41] J. Wu, J. Du, L. Liu, Q. Li, W. Rong, L. Wang, Y. Wang, M. Zang, Z. Wu, Y. Zhang, C. Qu, Elevated pretherapy serum IL17 in primary hepatocellular carcinoma patients correlate to increased risk of early recurrence after curative hepatectomy, PloS one, 7 (2012) e50035.

[42] H. Yabushita, M. Shimazu, H. Yamada, K. Sawaguchi, M. Noguchi, M. Nakanishi, M. Kawai, Occult lymph node metastases detected by cytokeratin immunohistochemistry predict recurrence in node-negative endometrial cancer, Gynecologic oncology, 80 (2001) 139-144. 
[43] R.B. Yang, C.K. Ng, S.M. Wasserman, L.G. Komuves, M.E. Gerritsen, J.N. Topper, A novel interleukin-17 receptor-like protein identified in human umbilical vein endothelial cells antagonizes basic fibroblast growth factor-induced signaling, The Journal of biological chemistry, 278 (2003) 33232-33238.

[44] W. Yang, A. Yan, T. Zhang, J. Shao, T. Liu, X. Yang, W. Xia, Y. Fu, Thromboxane A2 Receptor Stimulation Enhances Microglial Interleukin-1beta and NO Biosynthesis Mediated by the Activation of ERK Pathway, Frontiers in aging neuroscience, 8 (2016) 8.

[45] Z. Yao, W.C. Fanslow, M.F. Seldin, A.M. Rousseau, S.L. Painter, M.R. Comeau, J.I. Cohen, M.K. Spriggs, Herpesvirus Saimiri encodes a new cytokine, IL-17, which binds to a novel cytokine receptor, Immunity, 3 (1995) 811-821.

[46] P. Ye, P.B. Garvey, P. Zhang, S. Nelson, G. Bagby, W.R. Summer, P. Schwarzenberger, J.E. Shellito, J.K. Kolls, Interleukin-17 and lung host defense against Klebsiella pneumoniae infection, American journal of respiratory cell and molecular biology, 25 (2001) 335-340.

[47] B. Zhang, J. Wang, W. Liu, Y. Yin, D. Qian, H. Zhang, B. Shi, C. Li, J. Zhu, L. Zhang, L. Gao, C. Wang, Cytokeratin 18 knockdown decreases cell 
migration and increases chemosensitivity in non-small cell lung cancer, Journal of cancer research and clinical oncology, 142 (2016) 2479-2487. [48] Q. Zhang, S. Liu, K.R. Parajuli, W. Zhang, K. Zhang, Z. Mo, J. Liu, Z. Chen, S. Yang, A.R. Wang, L. Myers, Z. You, Interleukin-17 promotes prostate cancer via MMP7-induced epithelial-to-mesenchymal transition, Oncogene, 36 (2017) 687-699.

[49] X. Zhu, L.A. Mulcahy, R.A. Mohammed, A.H. Lee, H.A. Franks, L. Kilpatrick, A. Yilmazer, E.C. Paish, I.O. Ellis, P.M. Patel, A.M. Jackson, IL-17 expression by breast-cancer-associated macrophages: IL-17 promotes invasiveness of breast cancer cell lines, Breast cancer research : BCR, 10 (2008) R95. 


\section{Figure Legends}

Fig1. Identification of IL-17RB as a potential target for the prevention of lung cancer progression. (A) Kaplan-Meier analysis of OS, according to the expression of IL17A, IL17B, IL17C, IL17D, IL17E and IL17F, using publicly available lung cancer online microarray datasets software. HR=hazard ratio. (B) Relative IL17RA (228685_at, 220101_at and 229295_at), IL17RB (219255_x_at and 224156_x_at) and IL17RE (236186_x_at) mRNA levels in patients with lung cancer adenocarcinoma in the GSE31210 datasets. The significance is determined by Student $t$ test. ${ }^{*}, P<0.01$.

Fig2. IL-17RB is overexpressed in lung cancer and is correlated with poor survival. (A) Quantification of IL-17RB expression in 40 paired lung cancer specimens and significance determined by Student $t$ test. ${ }^{*}, P<0.05$. (B) IL-17RB expression in lung cancer tissues. (C) Kaplan-Meier survival curves for disease-free survival (DFS) and overall survival (OS) in lung cancer patients according to IL-17RB expression, as determined by IHC. (D) HRs for the risk of death, as determined by multivariable Cox regression, in lung cancer patients. 
Fig3. IL-17RB enhances migration/invasion and metastasis in lung cancer. (A) Top, Western blot analysis of IL-17RB expression in lung cancer cell lines. Bottom, invasive abilities of lung cancer cell lines. (B) Correlation between IL-17RB expression and invasion ability in lung cancer cell lines. (C) IL-17RB knockdown on migration/invasion potential of A549 and CL1-5 lung cancer cells. Top, Western blot analysis of IL-17RB expression in IL-17RB-knockdown cells vs. shluc control cells. Bottom, A549 and CL1-5 migration and invasion ability after IL-17RB knockdown. The data are presented as the mean \pm SD and the significance is determined by one way ANOVA followed by Tukey's post hoc test. ${ }^{*}, P<0.05$; ${ }^{* *}, P<0.01$. (D) IL-17RB overexpression on migration/invasion potential of CL1-0 and H441 lung cancer cells. Top, Western blot analysis of IL-17RB expression after IL-17RB overexpression vs. vector control treatment. Bottom, CL1-0 and H441 migration and invasion ability after IL-17RB overexpression. The data are presented as the mean \pm SD and the significance is determined by Student $t$ test. ${ }^{*}, P<0.01$. (E) Representative image of H\&E-stained lung tissues from mice intravenously injected with H441-vector or H441-IL17RB $(n=6)$. The data are presented as the mean \pm SD and the significance is determined by Student $t$ test. ${ }^{* *}, P<0.01$. (F) Representative images of CK18 expression in the lymph 
nodes of vector control mice and IL-17RB-overexpressing mice. The data are presented as the mean \pm SD and the significance is determined by Student $t$ test. ${ }^{* *}, P<0.01$.

Fig4. IL-17RB promotes invasion/migration through the ERK/ $\beta$-catenin pathway. (A) Top, phosphorylation kinase array assay in IL-17RB-overexpressing H441 cells. Bottom, phosphorylation levels of candidate proteins relative to the control. (B) Western blot analysis of candidate kinases in IL-17RB-overexpressing H441 cells. (C) Migration and invasion ability in CL1-0/IL-17RB and H441/IL-17RB cells after treatment with the MEK/ERK inhibitor PD98059 (25 $\mu \mathrm{M})$. The data are presented as the mean $\pm \mathrm{SD}$ and the significance is determined by one way ANOVA followed by Tukey's post hoc test. ${ }^{*}, P<0.05 ;{ }^{*}, P<0.01$. (D) Western blot analysis of IL-17RB, ERK, GSK3 $\beta$ and $\beta$-catenin expression in CL1-0/IL-17RB and H441/IL-17RB cells after treatment with the MEK/ERK inhibitor PD98059 (25 $\mu M)$. (E) Comparison of IL-17RB, ERK, GSK3 $\beta$ and $\beta$-catenin expression between IL-17RB-knockdown A549 and CL1-5 cells and shluc control cells.

Fig5. IL-17RB expression is correlated with Snail/Twist expression in 
lung cancer tissues. (A) Western blot analysis of IL-17RB and EMT markers in CL1-0/IL-17RB and H441/IL-17RB cells after treatment with the MEK/ERK inhibitor. (B) The expression of EMT markers in A549 and CL1-5 lung cancer cells after IL-17RB knockdown. (C) Representative images of IL-17RB and Snail IHC staining in lung cancer tissues. (D) Representative images of IHC analysis for IL-17RB and Twist in lung cancer tissues. (E and F) Quantification of IL-17RB and Snail (E)/Twist (F) expression by IHC analysis of lung cancer tissues. The number ( $\mathrm{n}$ ) of specimens is shown at the top of each column. *, $P<0.05$. (G) Analysis of the correlation between $I L-17 R B$ expression and Snail/Twist mRNA expression using GSE3141 datasets.

\section{Fig6. IL-17RB enhanced lung cancer migration/invasion in a}

IL-17B-mediated manner. (A) Top, Western blot analysis of IL-17RB expression following treatment with IL-17B $(12.5 \mathrm{ng} / \mathrm{mL})$. Bottom, relative expression of IL-17RB in IL-17B treated cells relative to untreated cells and the significance is determined by Student $t$ test. ${ }^{*}, P<0.05$. (B) IL-17B levels in IL-17RB-overexpressing cells after treatment with the MEK/ERK inhibitor PD98059. IL-17B concentrations in conditioned medium were quantified by ELISA. The data are shown as the mean \pm SD and the significance is 
determined by one way ANOVA followed by Tukey's post hoc test. ${ }^{*}, P<0.05$. (C) Migration/invasion ability in CL1-0/LL-17RB cells and H441/IL-17RB cells after pretreatment with an IL-17B-neutralizing antibody $(4 \mu \mathrm{g} / \mathrm{mL})$ or control $\operatorname{lgG}(4 \mu \mathrm{g} / \mathrm{mL})$ for 48 hours. The data are shown as the mean $\pm \mathrm{SD}$ and the significance is determined by one way ANOVA followed by Tukey's post hoc test. ${ }^{*}, P<0.05,{ }^{* *}, P<0.01$. (D) IL-17B levels in IL-17RB-overexpressing CL1-0 cells after IL-17B knockdown. Left, IL-17B levels were measured by ELISA. The data are shown as the mean $\pm S D$ and the significance is determined by one way ANOVA followed by Tukey's post hoc test. ${ }^{* *}, P<0.01$. Right, Western blot analysis of IL-17RB, p-ERK1/2 and ERK expression following IL-17B knockdown. (E) Western blot analysis of p-ERK1/2, ERK, IL-17B and IL-17RB expression following treatment with the ERK activator U46619 (10 $\mu \mathrm{M})$ or the MEK inhibitor PD98059 (25 $\mu \mathrm{M})$. (F) IL17B levels, determined by ELISA, in the serum of mice with lung metastasis of IL-17RB-overexpressing cancer cells and vector control-treated mice $(n=5)$. The data are shown as the mean \pm SD and the significance is determined by Student $t$ test. ${ }^{* *}, P<0.01$. (G) $I L-17 B$ and IL-17RB mRNA expression correlation analysis was performed using GSE3141 datasets. (H) Model showing that IL-17B-IL-17RB signaling induced ERK phosphorylation, leading to up-regulation of $\beta$-catenin, Snail, Twist, and 
cell invasion and metastasis in lung cancer. 
A

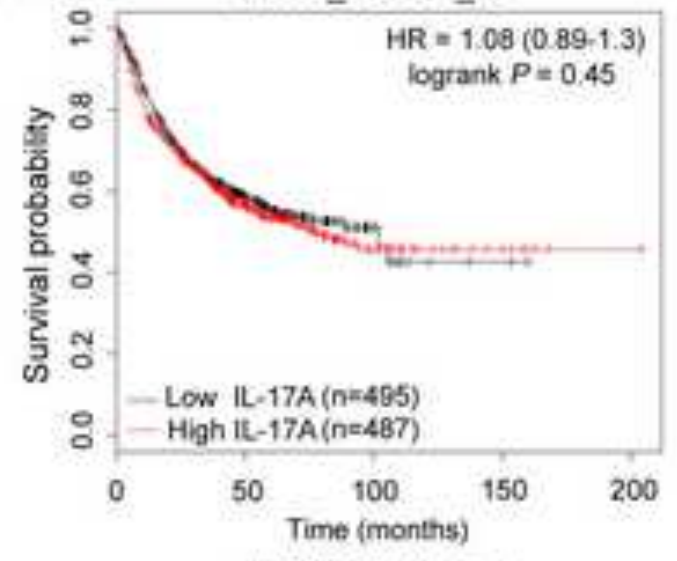

IL.17D_227401_at

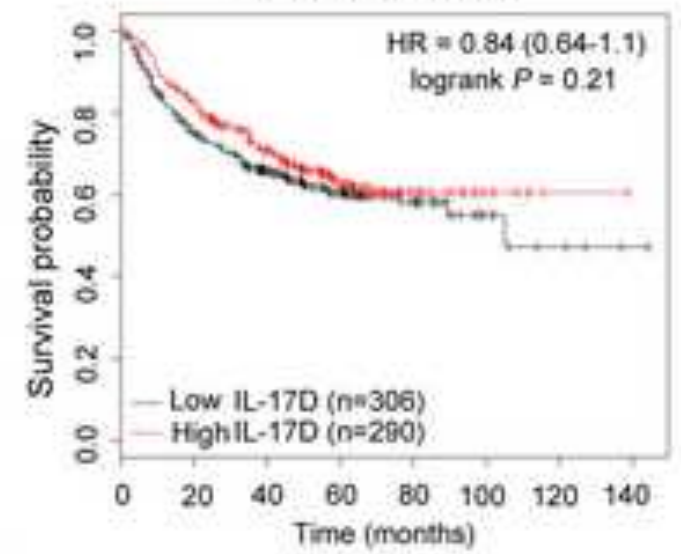

B H17RA_228685_at U17RA_229101_at

IC17RA_229101_at
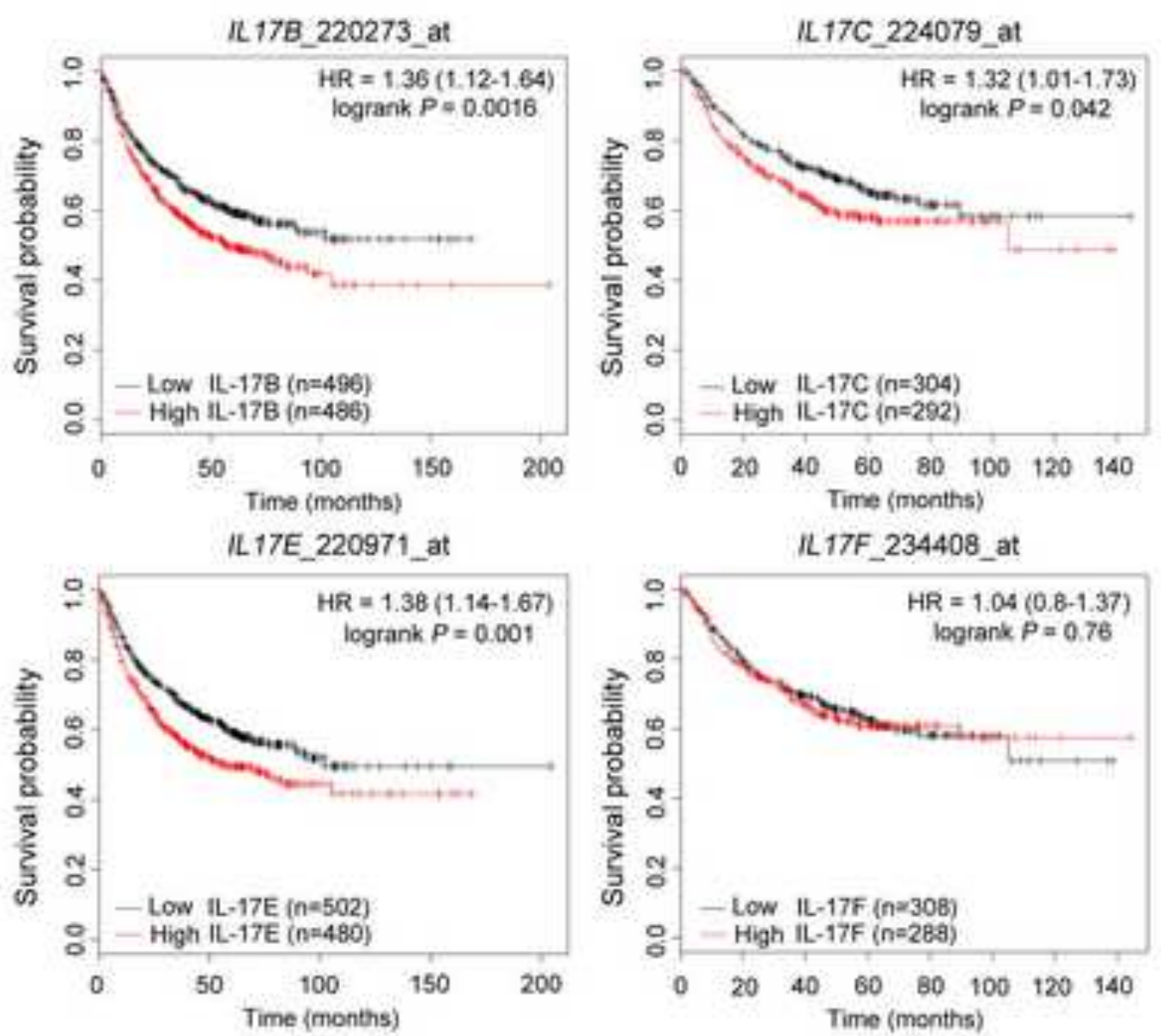

IL 17RA_229295_at UL17RB_219255_x_at

II 17RB_224158_x_at UL.17RE_238186_X_at
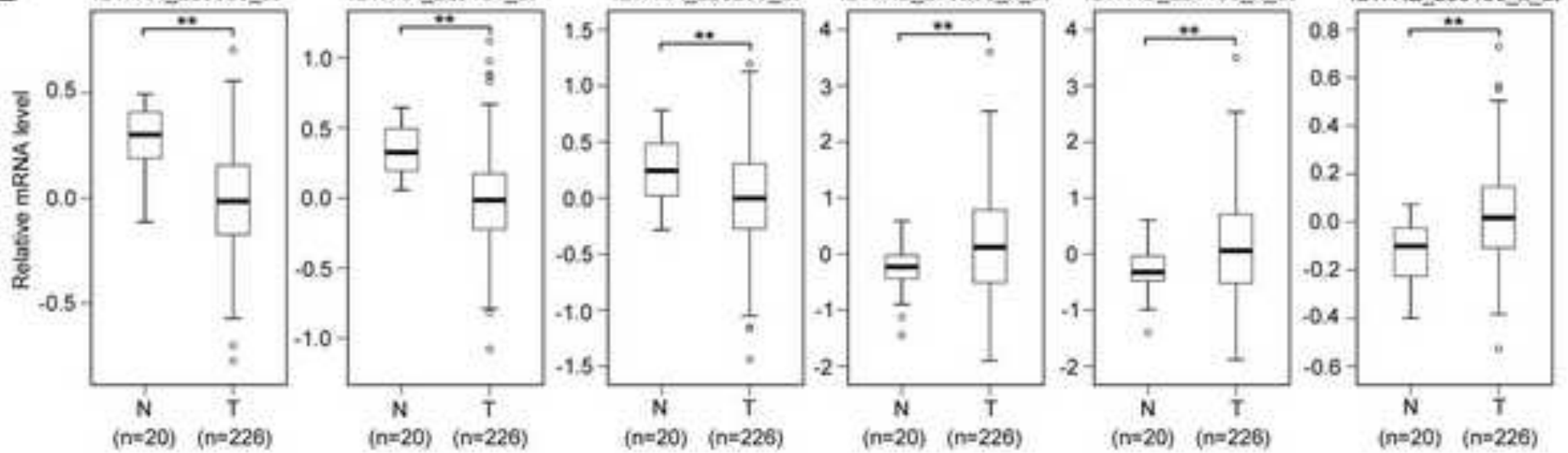


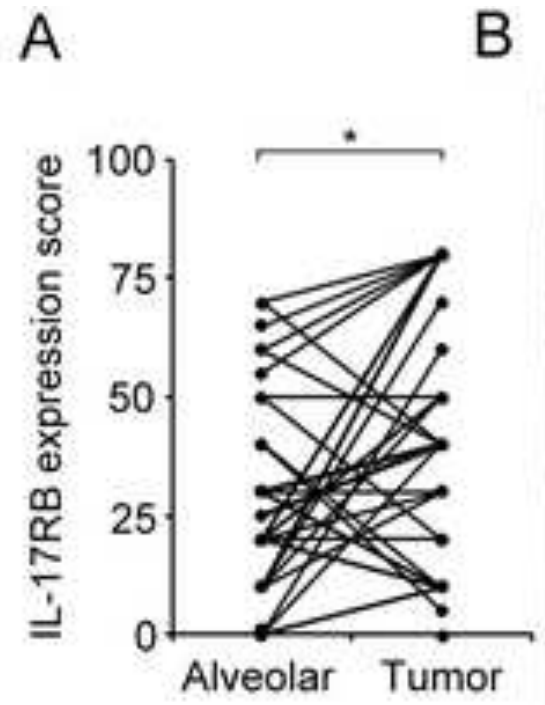

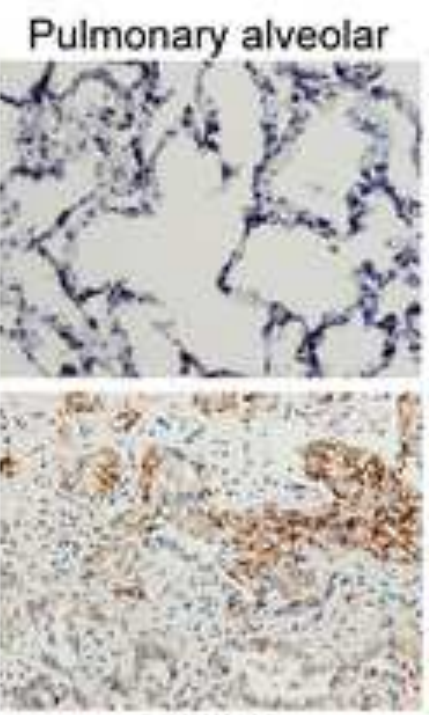

Score2

C

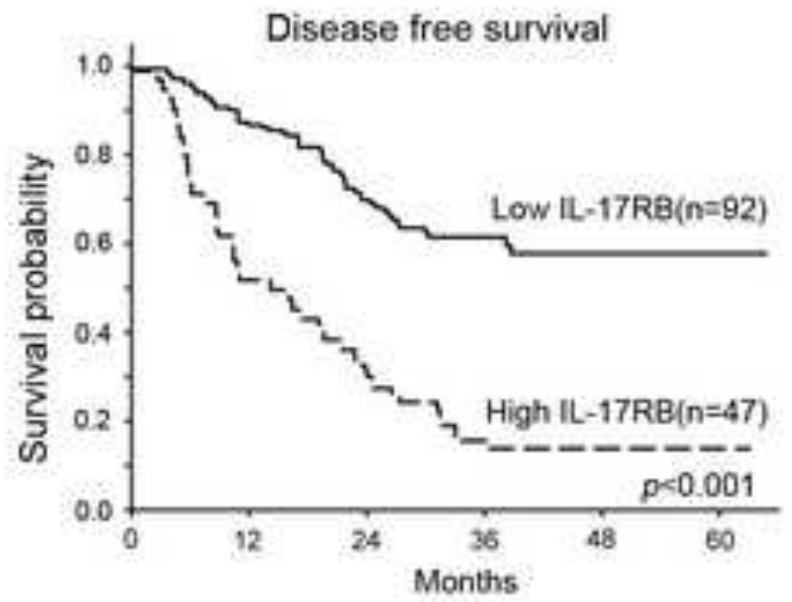

D

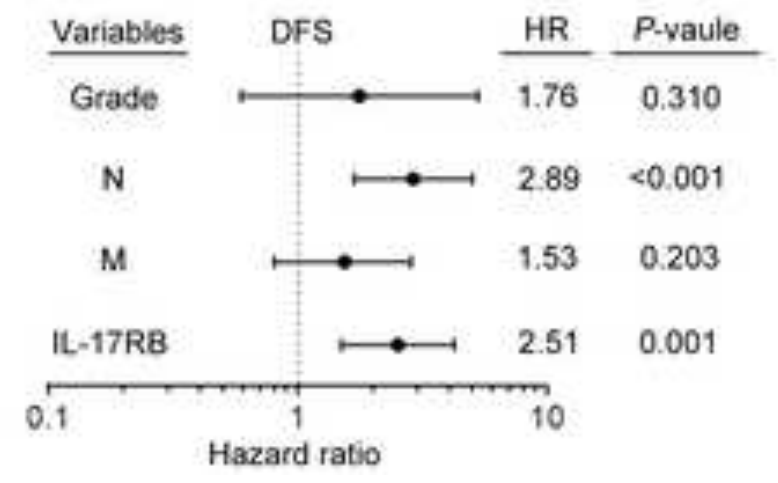

Negative

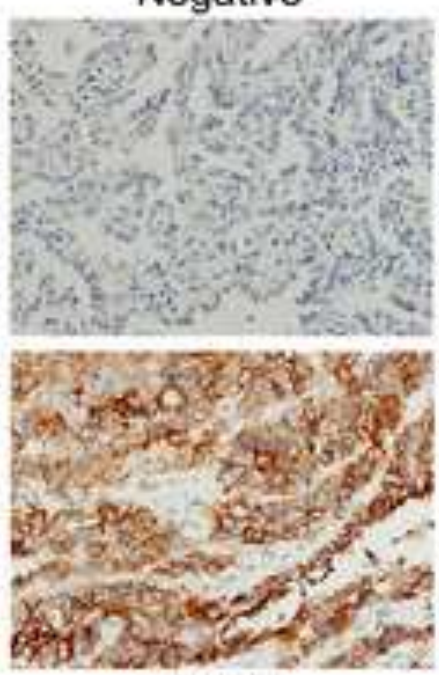

Score3
Score1

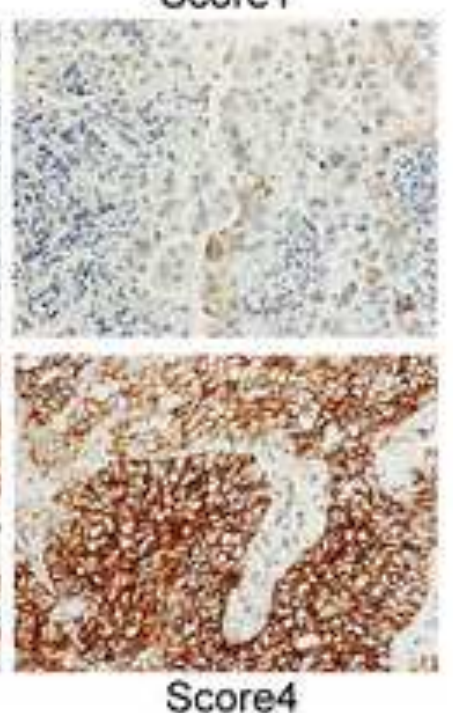

Overall survival
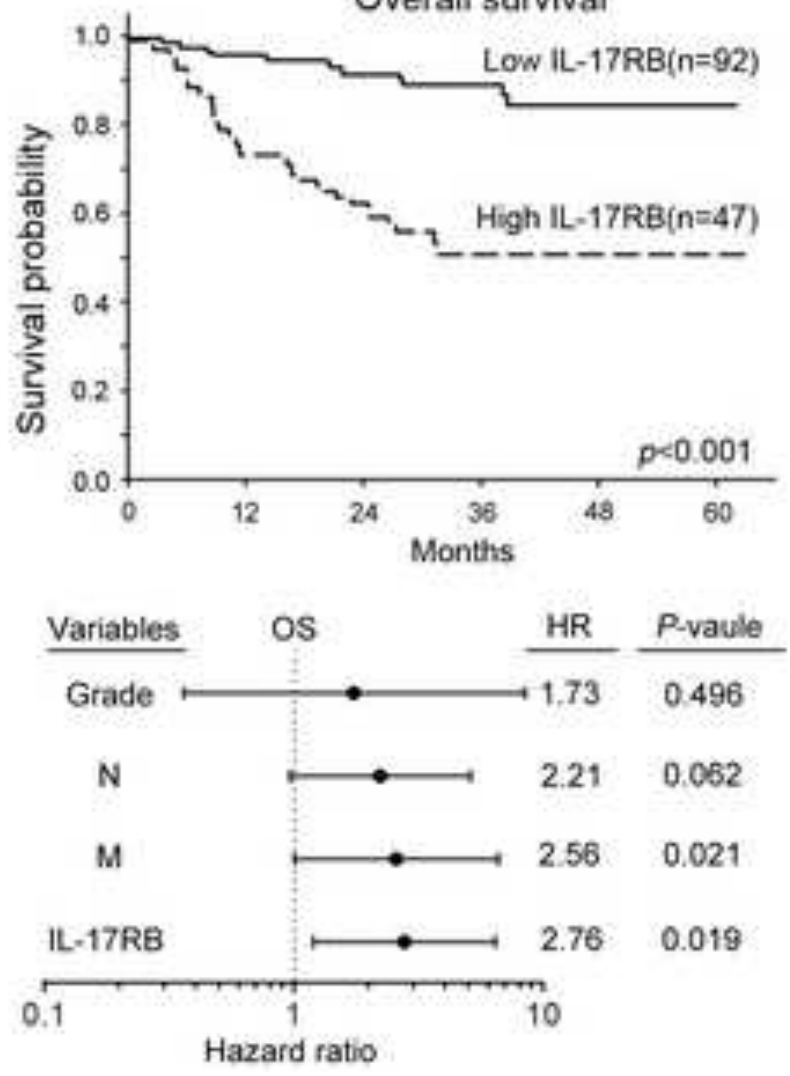


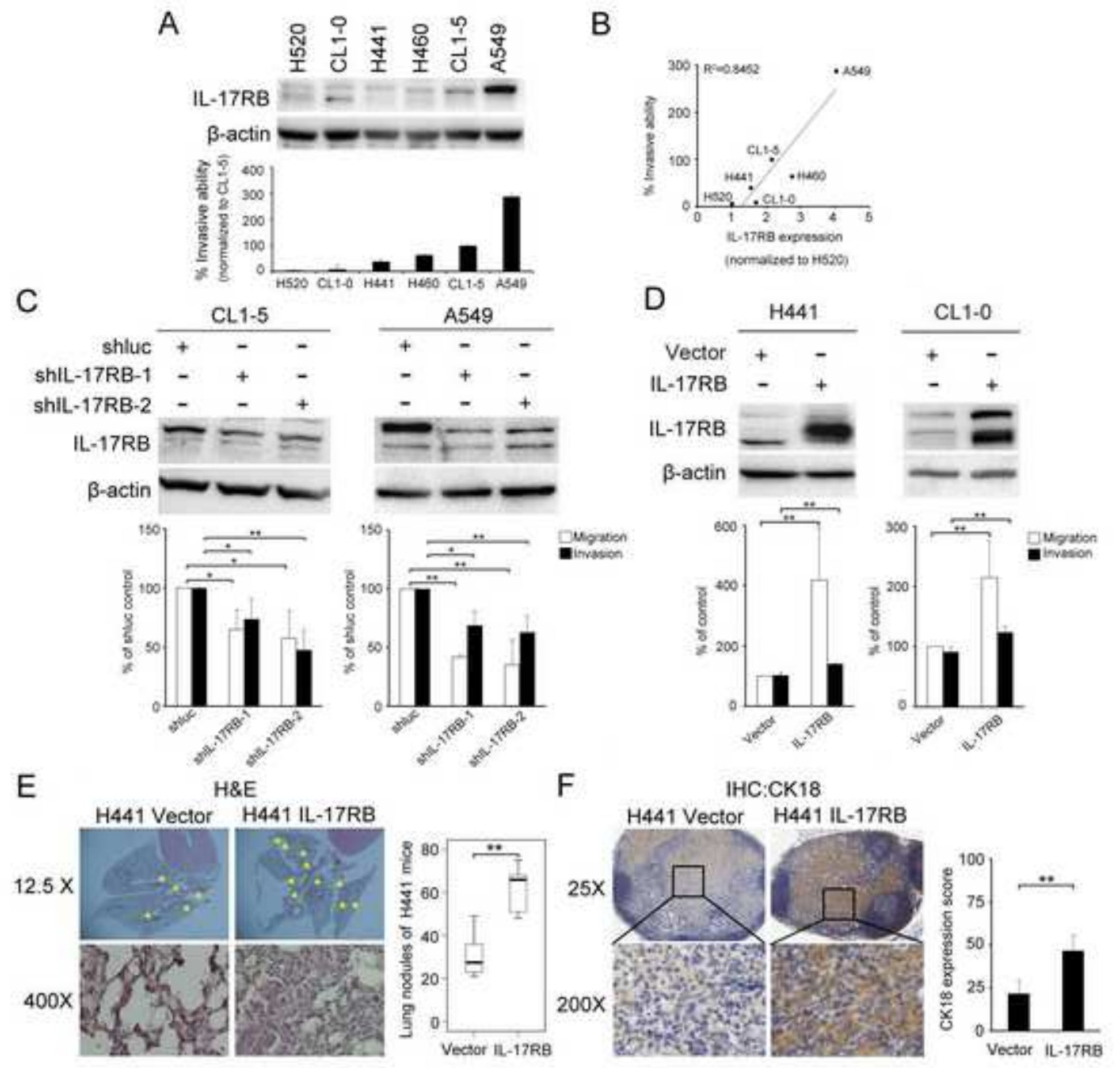

Vector 1L-17RB 
A
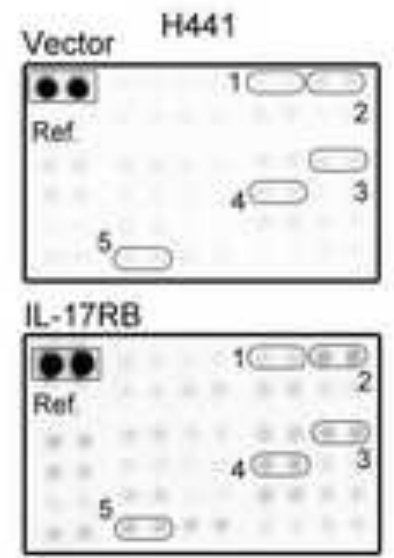

1.p-ERK 2p-GSK3 3. $\beta$-catenin 4 p AMPK 5.p-ChK2

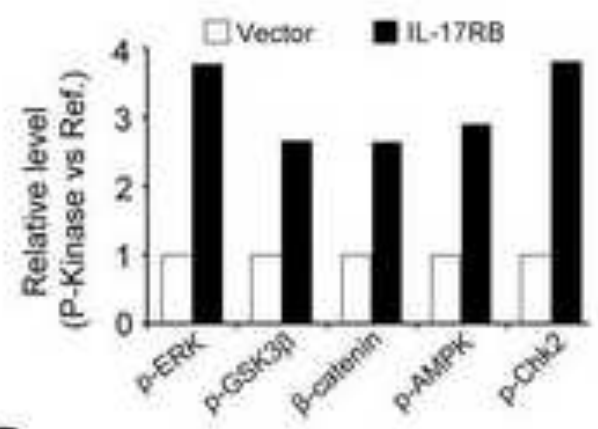

D

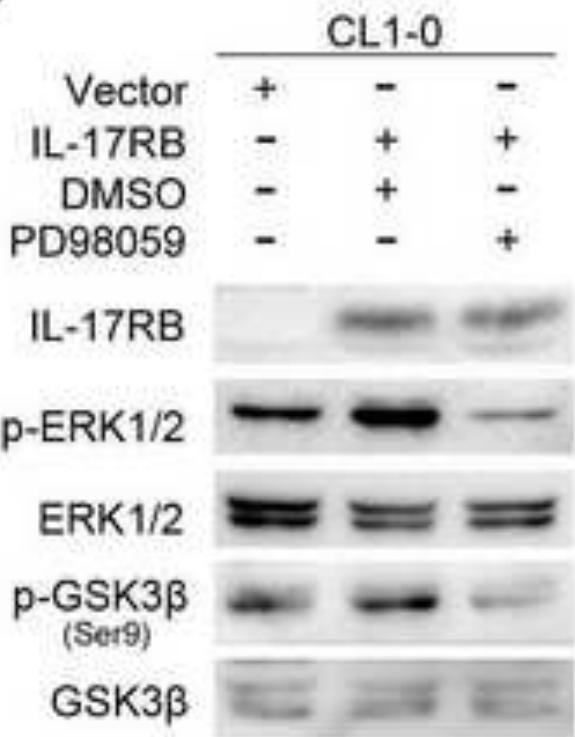

$\beta$-catenin

$\beta$-actin
B

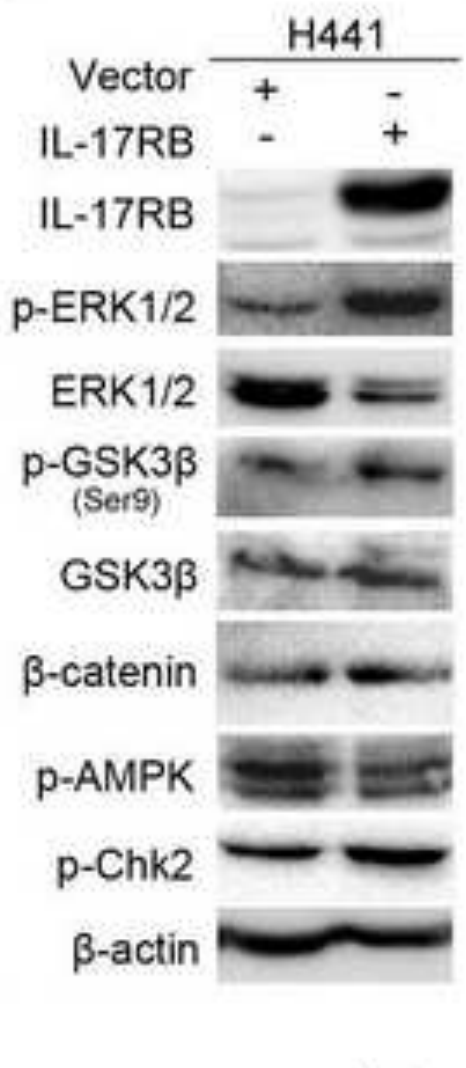

E
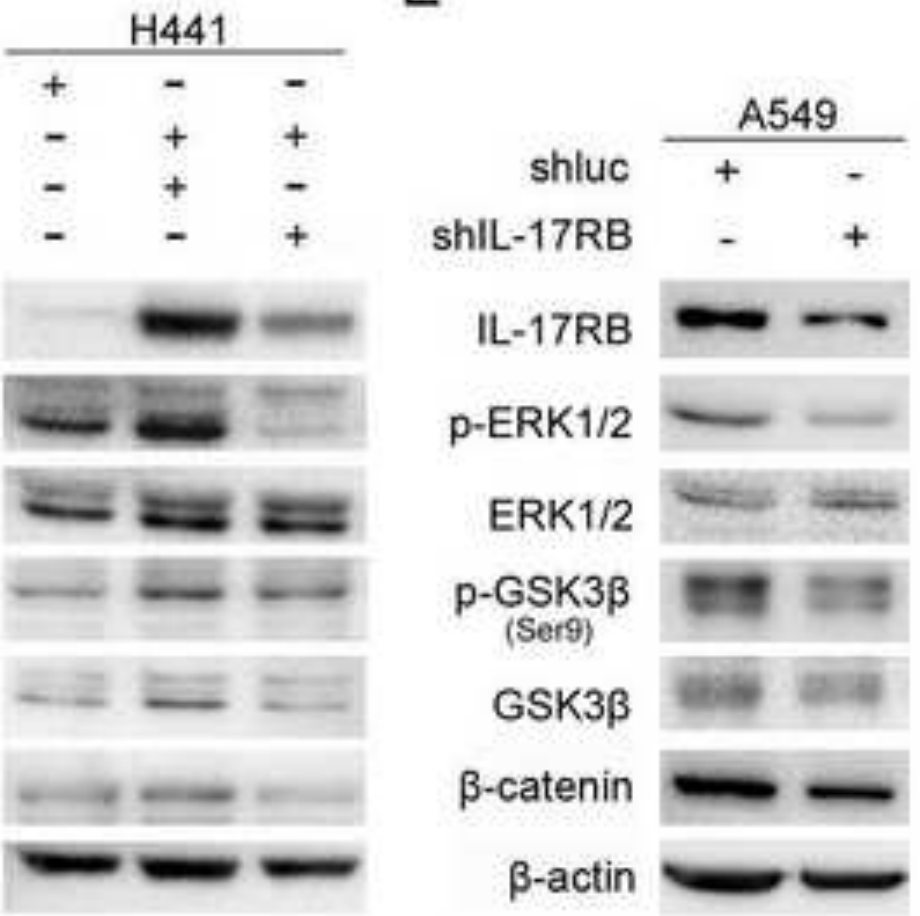

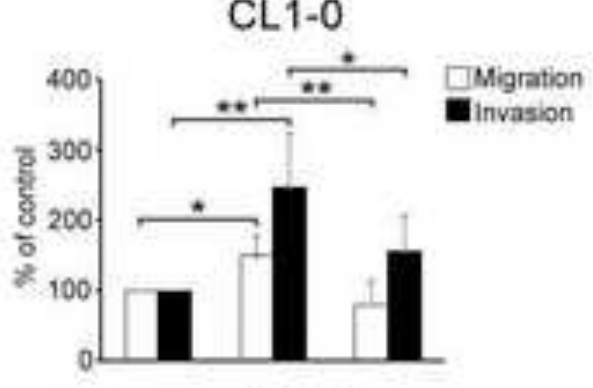

$\mathrm{H} 441$

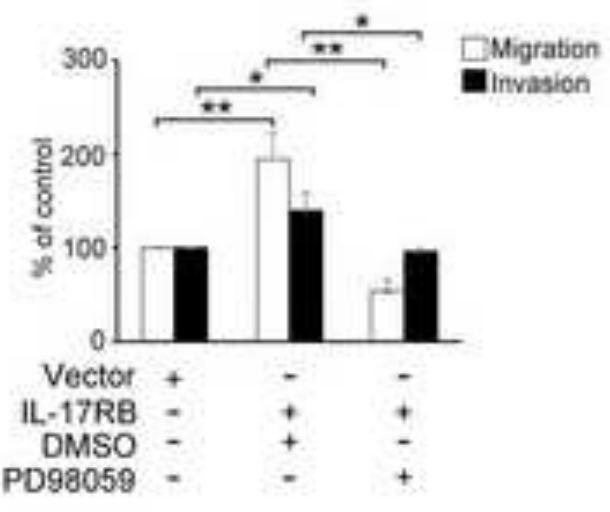


A

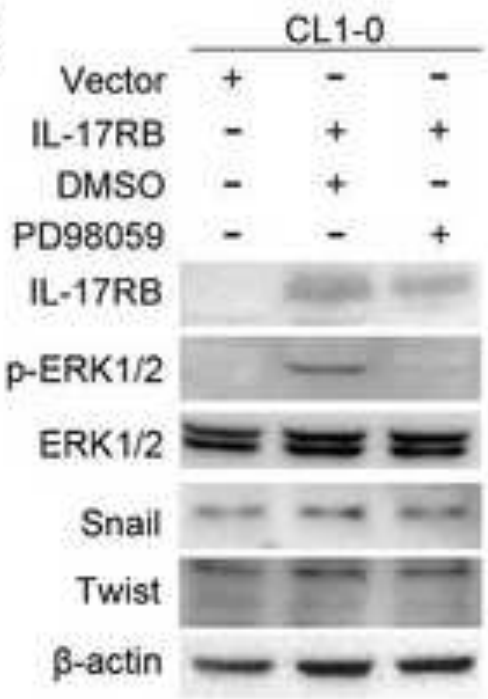

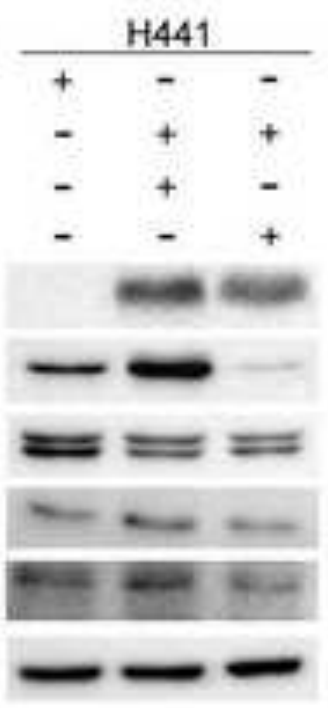

C

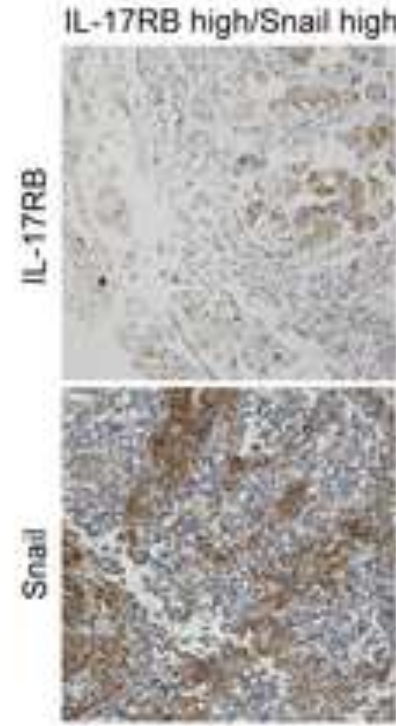

IL-17RB lowiSnail low
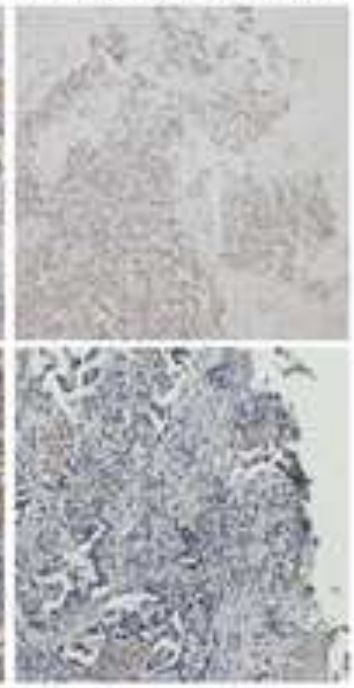

B

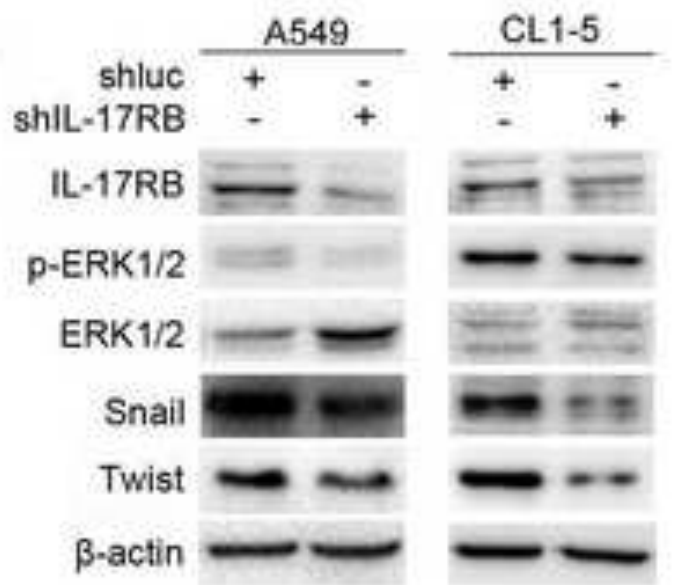

D

IL-17RB high/Twist high IL-17RB low/Twist low
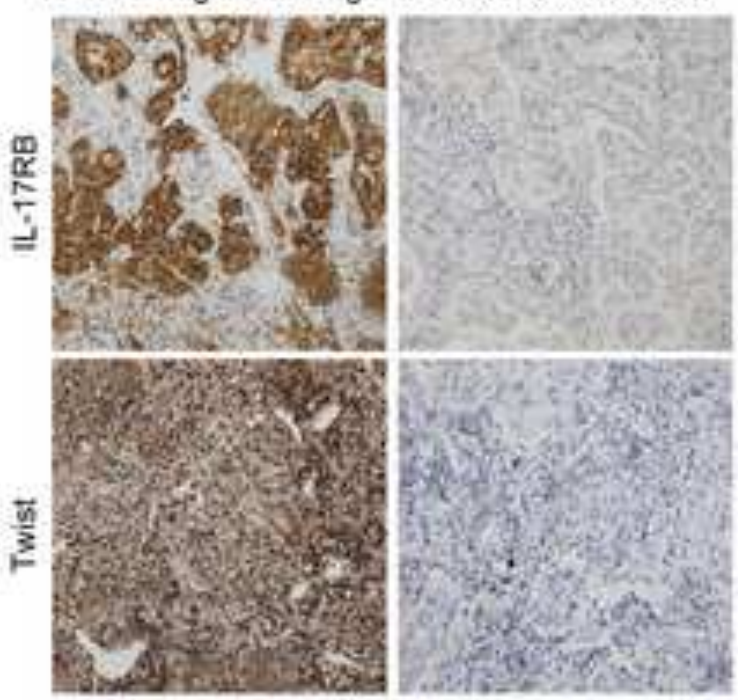

E

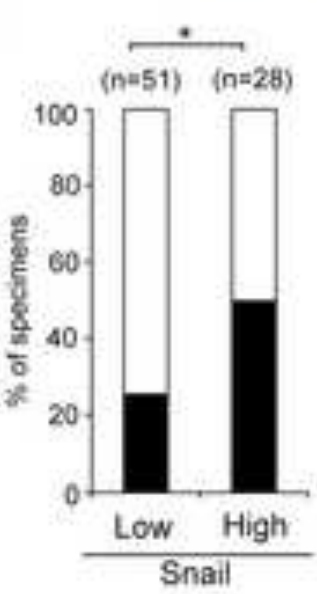

-High LL-17RB expression DLow IL-17RB expression
$\mathrm{F}$

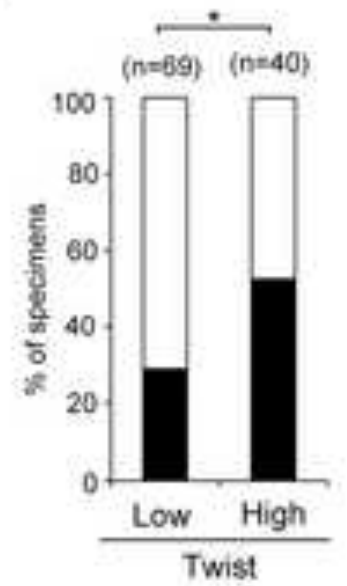

G
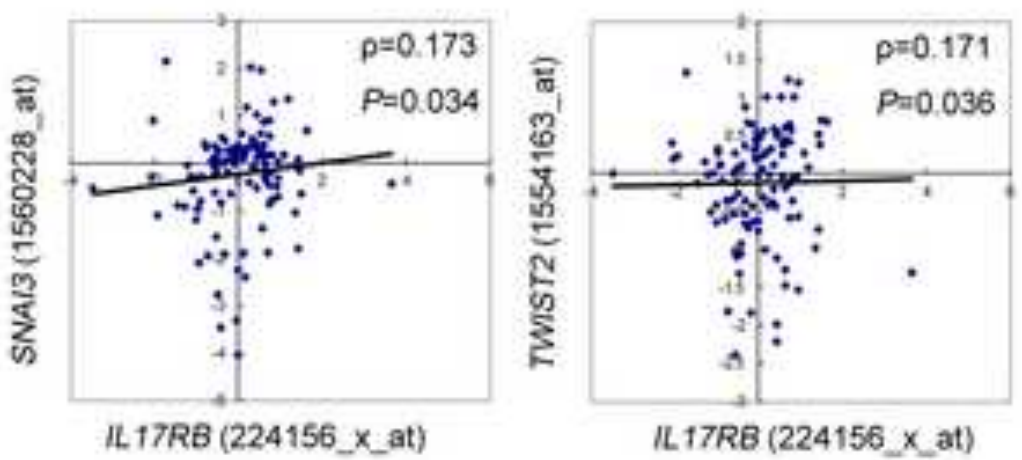

IL T7RB (224156 $\times$ at)

- High IL-17RB expression DLow IL-17RB expression 


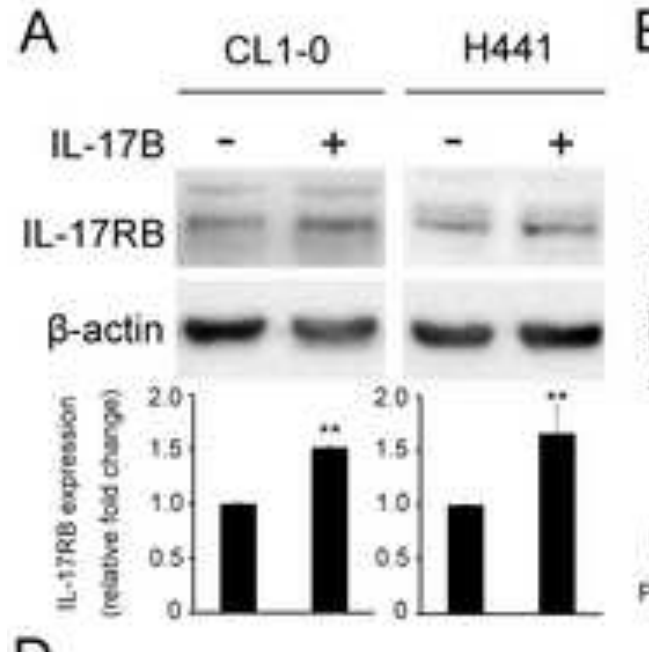

D

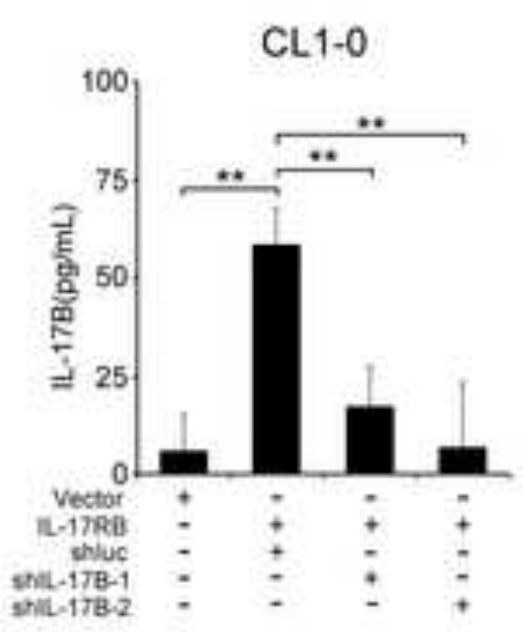

F

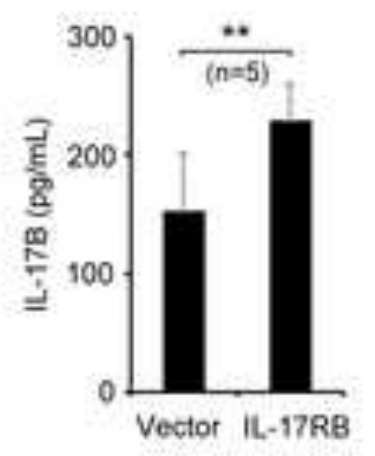

B

CL1-0
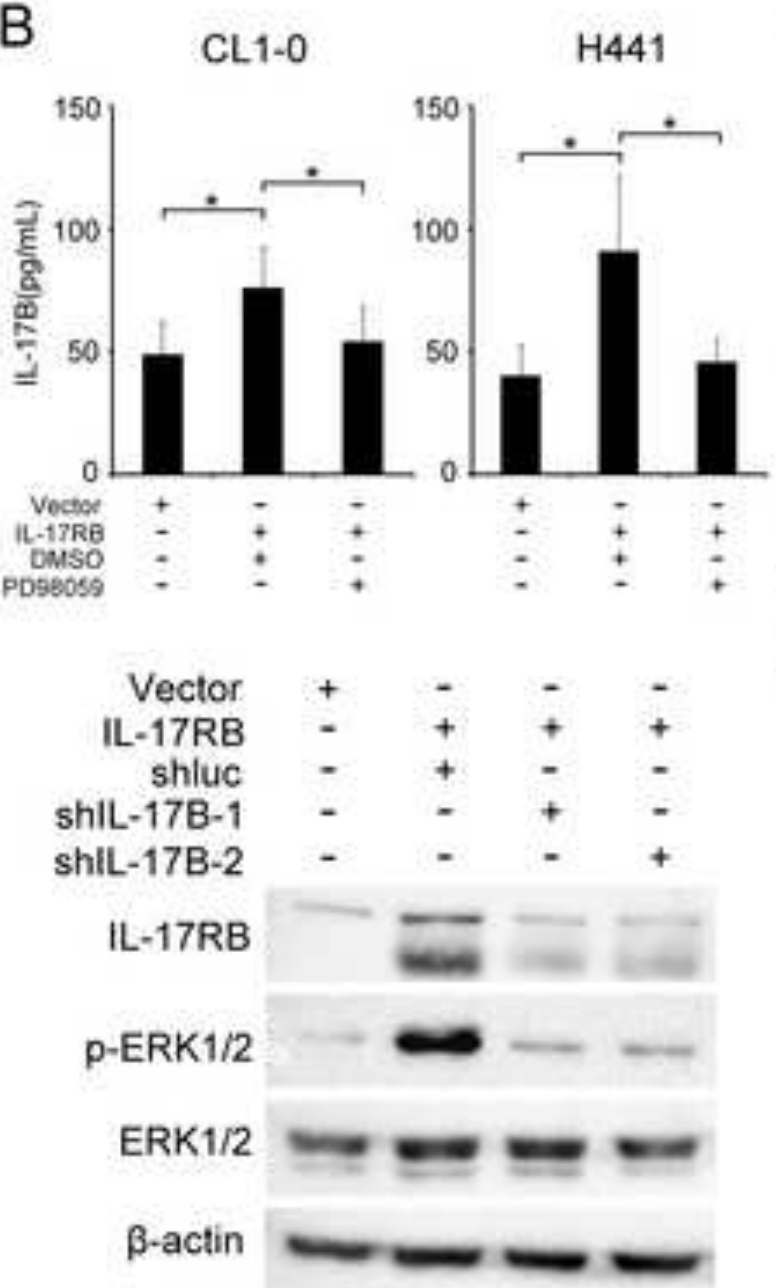

G

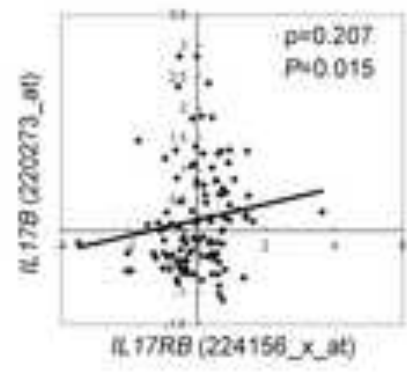

C CL1-0
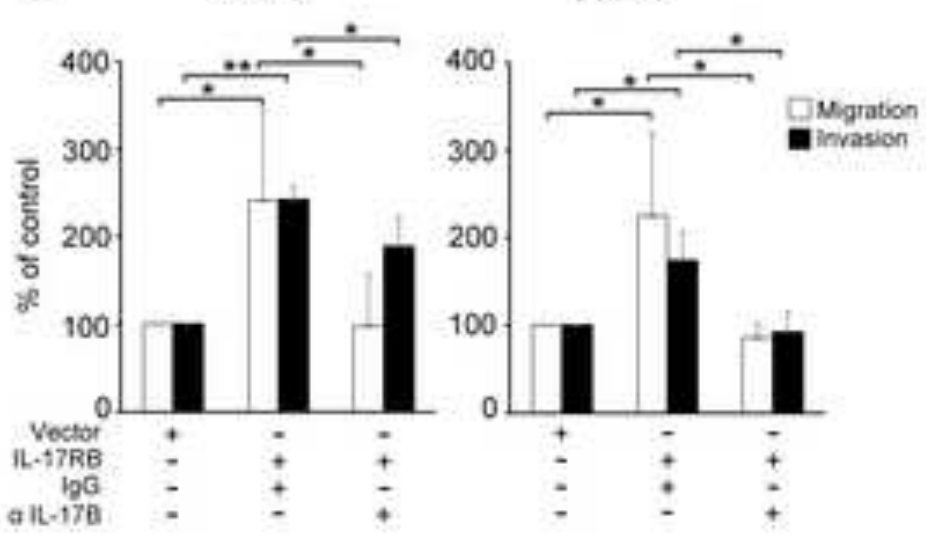

E

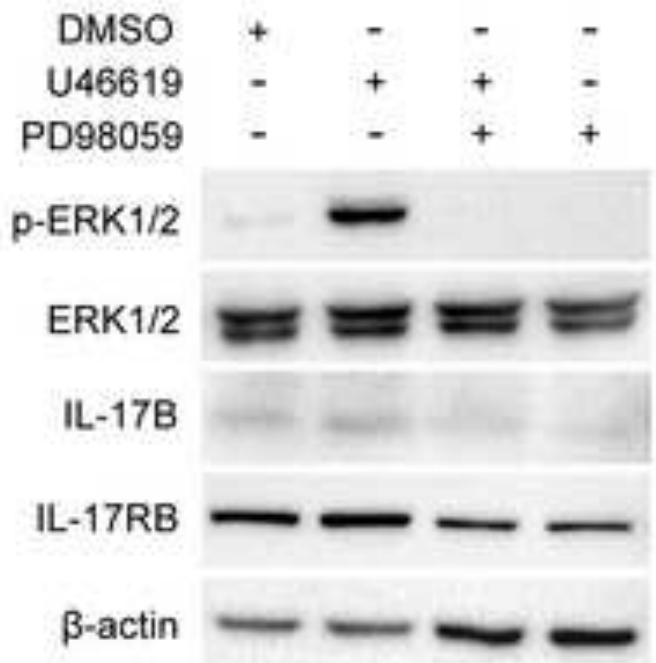

H

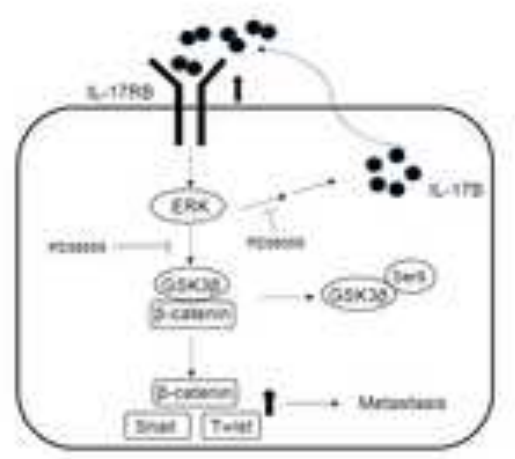


Table 1. Association between IL-17RB expression and clinicopathological characteristics in lung cancer.

\begin{tabular}{|c|c|c|c|c|c|}
\hline \multirow[b]{3}{*}{ Variables } & \multirow[b]{3}{*}{ Item } & \multirow[b]{3}{*}{ Patient No. } & \multicolumn{2}{|c|}{ IL17-RB } & \multirow[b]{3}{*}{$p$ value $^{*}$} \\
\hline & & & Low $(1,2)$ & High $(3,4)$ & \\
\hline & & & No. & No. & \\
\hline & & 139 & 92 & 47 & \\
\hline \multirow[t]{2}{*}{ Age $(y)$} & $\leq 70$ & 93 & 61 & 32 & 0.833 \\
\hline & $>70$ & 46 & 31 & 15 & \\
\hline \multirow[t]{2}{*}{ Sex } & Female & 47 & 34 & 13 & 0.273 \\
\hline & Male & 92 & 58 & 34 & \\
\hline \multirow[t]{3}{*}{ Grade } & 1 & 26 & 20 & 6 & 0.093 \\
\hline & ॥ & 99 & 66 & 33 & \\
\hline & III & 14 & 6 & 8 & \\
\hline \multirow[t]{2}{*}{ T status } & $\mathrm{T} 1 / \mathrm{T} 2$ & 113 & 74 & 39 & 0.716 \\
\hline & $\mathrm{T} 3 / \mathrm{T} 4$ & 26 & 18 & 8 & \\
\hline \multirow[t]{2}{*}{$\mathrm{N}$ status } & Negative (N0) & 84 & 64 & 20 & $0.002^{*}$ \\
\hline & $\begin{array}{l}\text { Positive } \\
\text { (N1/N2/N3) }\end{array}$ & 55 & 28 & 27 & \\
\hline \multirow[t]{2}{*}{ M status } & Negative & 119 & 83 & 36 & $0.030^{*}$ \\
\hline & Positive & 20 & 9 & 11 & \\
\hline \multirow[t]{2}{*}{ Performance } & 0 & 105 & 73 & 32 & 0.144 \\
\hline & 1 & 34 & 19 & 15 & \\
\hline \multirow[t]{3}{*}{ Smoking status } & Never & 85 & 62 & 23 & 0.094 \\
\hline & Former & 25 & 13 & 12 & \\
\hline & Current & 29 & 17 & 12 & \\
\hline
\end{tabular}

